\title{
A LIE THEORETIC APPROACH TO RENORMALIZATION
}

\author{
KURUSCH EBRAHIMI-FARD, JOSÉ M. GRACIA-BONDÍA, AND FRÉDÉRIC PATRAS
}

\begin{abstract}
Motivated by recent work of Connes and Marcolli, based on the Connes-Kreimer approach to renormalization, we augment the latter by a combinatorial, Lie algebraic point of view. Our results rely both on the properties of the Dynkin idempotent, one of the fundamental Lie idempotents in the theory of free Lie algebras, and on properties of Hopf algebras encapsulated in the notion of associated descent algebras. Besides leading very directly to proofs of the main combinatorial aspects of the renormalization procedures, the new techniques give rise to an algebraic approach to the Galois theory of renormalization. In particular, they do not depend on the geometry underlying the case of dimensional regularization and the Riemann-Hilbert correspondence. This is illustrated with a discussion of the BPHZ renormalization scheme.
\end{abstract}

PACS 2006: 03.70.+k; 11.10.Gh; 02.10.Hh; 02.10.Ox

Keywords: renormalization; beta function; dimensional regularization; BPHZ scheme; free Lie algebra; Hopf algebra; Rota-Baxter relation; Dynkin operator; descent algebra; Lie idempotent

\section{Contents}

1. Introduction

2. Notational conventions

3. The Hopf algebra of characteristic functions

4. Logarithmic derivatives and the Dynkin operator

5. Algebraic BWH decomposition of characters

6. On renormalization procedures

7. Locality and the Connes-Kreimer beta function

8. Through the prism of other renormalization schemes I

9. Through the prism of other renormalization schemes II

\section{INTRODUCTION}

From its inception, renormalization theory in perturbative quantum field theory (pQFT) had a combinatorial flavour, as well as an analytic one. The former manifests itself in the self-similarity of Feynman graphs, the building blocks of pQFT. The intricate combinatorics of extracting and combining subgraphs, required in the renormalization process, is encoded in the Bogoliubov recursion, respectively its solution via Zimmermann's forest formula $[13,15$, $41,52,53]$.

Kreimer's discovery of a Hopf algebra structure underlying Bogoliubov and Zimmermann's formulae and illuminating their internal structure [36] was the starting point of a new approach in the field. Then the Connes-Kreimer decomposition à la Birkhoff-Wiener-Hopf (BWH) of 
Feynman rules [18] captured the process of renormalization in pQFT in the framework of dimensional regularization (DR) with the minimal subtraction (MS) scheme. Further work by Connes, Kreimer and others has since then established various links between the BWH decomposition of characters in renormalizable quantum field theories and relevant mathematical topics, culminating recently in work by Connes and Marcolli on motivic Galois theory [21,22] and by Bloch, Esnault and Kreimer on the motivic nature of primitive Feynman graphs [6].

In the present work, largely motivated by [21], we return to the origin of the Connes-Kreimer theory and concentrate on algebraic features of renormalization relevant to pQFT, trying to unravel further fundamental properties of renormalization schemes by methods inspired on the classical theory of free Lie algebras (FLAs).

It has been known since the mid-nineties that many properties of FLAs, as exposed e.g. in $[9,47]$, can be lifted to general graded Lie algebras and their enveloping algebras. In other terms Lie theory is relevant to the study of arbitrary graded connected cocommutative or commutative Hopf algebras. In particular, the Solomon algebras of type $A_{n}$ [47, Chap. 9] act naturally on arbitrary graded connected commutative Hopf algebras [44, Thm. II.7].

The observation applies to the Hopf algebras of renormalization, yet it has not received the attention it deserves. Here we develop it systematically, considering abstract renormalization Hopf algebras $H$ and commutative target algebras $A$ of quantum amplitudes endowed with a Rota-Baxter operator. We show that some of the deepest combinatorial properties of renormalization schemes are codified in the composition with the Dynkin idempotent of Lie theory, and in its inverse map. We derive in particular from their study the properties of characters under locality assumptions for counterterms in renormalization and, in the process, establish that the data relevant to their computation are contained in the "beta function". The phenomenon is well known in pQFT; the Lie theoretic approach, however, provides a remarkably efficient way to deduce it from the locality assumptions.

Furthermore, the direct sum of Solomon algebras (the descent algebra) is naturally provided with a graded connected cocommutative Hopf algebra structure; the corresponding prounipotent group is naturally isomorphic to the universal group $U$ of the Connes-Marcolli Galois theory of renormalization. This isomorphism paves the way to an algebraic and combinatorial approach to the later theory. Some advantages of our method are: (i) It appears to be independent of the DR with MS prescription, applying in principle to any renormalization procedure which can be formulated in terms of a Rota-Baxter structure; (ii) Use of the Dynkin map explains naturally the coefficients of the universal singular frame of [21] - the same as in the Connes-Moscovici index formula [16] for the case of simple dimension spectrum.

The article is organized as follows. After settling some notation in the next section, we ponder in Section 3 the convolution algebra of linear maps $\operatorname{Lin}(H, A)$. It cannot be made into a Hopf algebra in general; but a suitable algebra of characteristic functions can. This is our playground; it encodes, at the Hopf algebra level, the properties of the group of $A$-valued characters of $H$. Section 4 is the heart of the paper: starting from a short survey on the Dynkin idempotent for cocommutative Hopf algebras, we establish the formal properties of its sibling in the commutative case, then introduce and exhaustively study the inverse Dynkin map. In particular, we show that the latter bijectively sends infinitesimal characters into characters of commutative connected Hopf algebras - applying in particular to the Hopf algebras of Feynman diagrams and rooted trees of renormalization theory and the corresponding Feynman rules. In Section 5 we recall the BWH decomposition of characters, choosing once again to obtain it algebraically from Rota-Baxter operator theory and the 'Baker-Campbell-Hausdorff $(\mathrm{BCH})$ recursion'. After that, our Lie theoretic machine is fully operational. 
In the rest of the paper, we show the relevance of that machine to pQFT. In Section 6 we briefly review some standard lore of renormalization and remind the reader of the dictionary between it and the Connes-Kreimer paradigm. Next we study in Section 7 the locality properties for dimensional regularization (DR) schemes by exploiting the properties of the Dynkin pair of maps, together with the BWH decomposition. The main results concerning the Connes-Kreimer beta function and the renormalization group (RG) in DR are rederived from direct computations in the algebra of characteristic functions; hopefully, the role of that beta function is thereby illuminated. Sections 8 and 9 are essays on the same question in other renormalization frameworks; in the second we invoke the BPHZ scheme of renormalization and exhibit the underlying Rota-Baxter algebra structure, exemplifying with the (GinzburgLandau-Wilson) $\varphi_{4}^{4}$ scalar model in Euclidean field theory.

To finish, in Section 10 we go back to the mathematical setting, trying to place our results in the 'great scheme of things' of combinatorial Hopf algebra theory. We show there how the Connes-Marcolli "motivic Galois group" of renormalization relates with FLAs as well as the theory of descent algebras. Together with the links between the same group and ConnesMoscovici's index theorem in noncommutative geometry, these new connections give further evidence for Connes' and Kreimer's - already much documented - claim that the divergences of pQFT do reveal the presence of deep mathematical structures.

\section{Notational COnVEntions}

Let $H=\bigoplus_{n=0}^{\infty} H_{n}$ be a graded connected commutative Hopf algebra (of finite type) over a field $k$ of characteristic zero; this is necessarily free as a commutative algebra [44, Prop. 4.3]. We write $\epsilon$ for the augmentation from $H$ to $H_{0}=k \subset H$ and $H^{+}$for the augmentation ideal $\bigoplus_{n=1}^{\infty} H_{n}$ of $H$. The identity map of $H$ is denoted $I$. The product in $H$ is written $\pi$ or simply by concatenation. The coproduct is written $\delta$; we use Sweedler's notation and write $h^{(1)} \otimes h^{(2)}$ for $\delta(h), h \in H_{n}$; or $\sum_{i=0}^{n} h_{i}^{(1)} \otimes h_{n-i}^{(2)}$ when the grading has to be taken into account. The usual restrictions apply, that is, $h^{(1)} \otimes h^{(2)}$ stands for a sum $\sum_{j \in J} h_{j}^{(1)} \otimes h_{j}^{(2)}$ and should not be interpreted as the tensor product of two elements of $H$. The same convention applies in forthcoming notation such as $h^{(1)} \otimes g^{(1)} \otimes h^{(2)} \otimes g^{(2)}$, that should be understood as $\sum_{j \in J} \sum_{k \in K} h_{j}^{(1)} \otimes g_{k}^{(1)} \otimes h_{j}^{(2)} \otimes g_{k}^{(2)}$, where $\delta(g)=\sum_{k \in K} g_{k}^{(1)} \otimes g_{k}^{(2)}$.

Graduation phenomena are essential for all our forthcoming computations, since in the examples of physical interest they incorporate information such as the number of loops (or vertices) in Feynman graphs, and the subdivergence structure, relevant for the RG. They are expressed by the action of the grading operator $Y: H \rightarrow H$, given by:

$$
Y(h)=\sum_{n \in \mathbb{N}} n h_{n} \quad \text { for } \quad h=\sum_{n \in \mathbb{N}} h_{n} \in \bigoplus_{n \in \mathbb{N}} H_{n} .
$$

We write $\left|h_{n}\right|:=n$. Notice that $Y$ is usually denoted by $D$ in the FLA literature. In the present article we stick to the notation most often used in the context of the Hopf algebra approach to renormalization $[17-19,21,25,30]$ and reserve $D$ for the Dynkin operator.

\section{The Hopf algebra of Characteristic Functions}

A character is a map $\gamma$ of unital algebras from $H$ to the base field $k$ :

$$
\gamma\left(h h^{\prime}\right)=\gamma(h) \gamma\left(h^{\prime}\right)
$$

It should be clear that the product on the right hand side is the one in $k$. We write $\gamma_{n}$ for the restriction of $\gamma$ to a map from $H_{n}$ to $k$. 
Let $A$ be a commutative $k$-algebra, with unit $1_{A}=\eta_{A}(1), \eta_{A}: k \rightarrow A$ and with product $\pi_{A}$, which we sometimes denote by a dot: $\pi_{A}(u \otimes v)=: u \cdot v$. The main examples we have in mind are $A=\mathbb{C}, A=\mathbb{C}\left[\left[\varepsilon, \varepsilon^{-1}\right]\right.$ and $A=H$. We extend now the definition of characters and call an ( $A$-valued) character of $H$ any algebra map from $H$ to $A$. In particular $H$-valued characters are simply algebra endomorphisms of $H$.

An infinitesimal character is a linear map $\alpha$ from $H$ to $k$ such that:

$$
\alpha\left(h h^{\prime}\right)=\alpha(h) \epsilon\left(h^{\prime}\right)+\epsilon(h) \alpha\left(h^{\prime}\right) .
$$

As for characters, we write $\alpha(h)=\sum_{n \in \mathbb{N}} \alpha_{n}\left(h_{n}\right)$. The same notational convention will be used in the sequel without further notice: $f_{n}$ stands for the restriction of an arbitrary map $f$ on $H$ to $H_{n}$. We remark that by the very definition of characters and infinitesimal characters $\gamma_{0}\left(1_{H}\right)=1$, that is $\gamma_{0}=\epsilon$, whereas $\alpha_{0}\left(1_{H}\right)=0$.

We extend as well in the obvious way the notion of infinitesimal characters to maps from $H$ to a commutative $k$-algebra $A$. We have now:

$$
\alpha\left(h h^{\prime}\right)=\alpha(h) \cdot e\left(h^{\prime}\right)+e(h) \cdot \alpha\left(h^{\prime}\right),
$$

where $e:=\eta_{A} \circ \epsilon$. They can be alternatively defined as $k$-linear maps from $H$ to $A$ with $\alpha_{0}=0$ that vanish on the square of the augmentation ideal of $H$. In particular, if $\alpha$ is an infinitesimal character, the linear map $\alpha^{\mid n}$, the restriction of which is 0 on all the graded components of $H$ excepted $H_{n}$, and $\alpha_{n}^{\mid n}=\alpha_{n}$, is also an infinitesimal character. Thus $\alpha$ decomposes as a sum of infinitesimal characters $\alpha=\sum_{n>0} \alpha^{\mid n}$. The vector space of infinitesimal characters, written $\Xi_{H}(A)$, or just $\Xi(A)$, decomposes accordingly as the direct product of its graded components: $\Xi(A)=\prod_{n \in \mathbb{N}^{*}} \Xi_{n}(A)$, where $\Xi_{n}(A)$ is the linear span of the $\alpha^{\mid n}$. Thus we regard $\Xi(A)$ as the natural 'topological' completion of the graded vector space $\oplus_{n \in \mathbb{N}^{*}} \Xi_{n}(A)$. In more detail, we consider the subspaces $\oplus_{i \geq n \in \mathbb{N}^{*}} \Xi_{i}(A)$ and the associated onto homomorphisms, and we take the inverse limit, whose elements are infinite series. This property we agree to abbreviate from now on to " $\Xi(A)$ is a graded vector space"; the sundry objects defined below are graded in that sense - that is, they are actually completions of graded vector spaces, completions of graded Lie algebras, and so on.

The space $\operatorname{Lin}(H, A)$ of $k$-linear maps from $H$ to $A, \operatorname{Lin}(H, A):=\prod_{n \in \mathbb{N}} \operatorname{Lin}\left(H_{n}, A\right)$, is naturally endowed with an algebra structure by the convolution product:

$$
f * g:=\pi_{A} \circ(f \otimes g) \circ \delta: \quad H \stackrel{\delta}{\rightarrow} H \otimes H \stackrel{f \otimes g}{\longrightarrow} A \otimes A \stackrel{\pi_{A}}{\longrightarrow} A .
$$

The unit for the convolution product is precisely $e: H \rightarrow A$. Especially when $A=H$, the convolution product provides various tools to deal with properties of characters, such as the logarithm of the identity, which is a projector on $H$ with kernel the square of the augmentation ideal. As a corollary, $A$-valued infinitesimal characters can be characterized as those maps $\alpha$ from $H$ to $A$ such that $\alpha_{n} \circ I^{* k}\left(h_{n}\right)=k \alpha_{n}\left(h_{n}\right)$, for any $k, n \in \mathbb{N}$. We refer the reader interested in a systematic study of these phenomena and of their connections to the classical structure theorems for Hopf algebras such as the Cartier-Milnor-Moore theorem to [12,43, 44].

The set $G_{H}(A)$ of characters, or just $G(A)$, is a group for the convolution product. The inverse is given explicitly by the formula:

$$
\gamma^{-1}=\left(e+\sum_{n \in \mathbb{N}^{*}} \gamma_{n}\right)^{-1}=e+\sum_{k \in \mathbb{N}^{*}}(-1)^{k}\left(\sum_{n \in \mathbb{N}^{*}} \gamma_{n}\right)^{* k} .
$$

The last sum is well-defined as a power series, since only a finite number of terms appear in each degree. We denote as usual by $S$ the convolution inverse of the identity map $I$ 
in $\operatorname{End}(H):=\operatorname{Lin}(H, H)$. Then $\gamma^{-1}=\gamma \circ S \in G(A)$; the reader unfamiliar with this identity can deduce it easily from the next lemma and other notions introduced soon.

Now, $\operatorname{Lin}(H, A)$ is not naturally provided with a Hopf algebra structure over the ground field $k$, except under particular assumptions on the target space $A$. For example, it is (up to the completion phenomena) a Hopf algebra if $A=k$. This follows from the usual argument to construct a Hopf algebra structure on the graded dual of a graded connected Hopf algebra of finite type. It is not when $A=k\left[\left[\varepsilon, \varepsilon^{-1}\right]\right.$, that is when the coefficient algebra $A$ is the field of Laurent series - an example relevant to renormalization. However, as will be shown below, a Hopf algebra structure can always be defined on certain remarkable spaces naturally related to $\operatorname{Lin}(H, A)$ and, most importantly in view of applications to pQFT, to the group of characters $G(A)$.

Lemma 3.1. Assume that, for given $\phi, \psi \in \operatorname{Lin}(H, A)$, there exist elements $\phi^{(1)} \otimes \phi^{(2)}$, respectively $\psi^{(1)} \otimes \psi^{(2)}$, in $\operatorname{Lin}(H, A) \otimes \operatorname{Lin}(H, A)$ such that, for any $h, h^{\prime} \in H$ :

$$
\phi^{(1)}(h) \cdot \phi^{(2)}\left(h^{\prime}\right)=\phi\left(h h^{\prime}\right) \quad \text { and } \quad \psi^{(1)}(h) \cdot \psi^{(2)}\left(h^{\prime}\right)=\psi\left(h h^{\prime}\right) ;
$$

then

$$
\phi * \psi\left(h h^{\prime}\right)=\left(\phi^{(1)} * \psi^{(1)}(h)\right) \cdot\left(\phi^{(2)} * \psi^{(2)}\left(h^{\prime}\right)\right) .
$$

Moreover, when $\psi \in \operatorname{End}(H)$ and $\phi \in \operatorname{Lin}(H, A)$, with the same hypothesis and $\psi^{(1)}, \psi^{(2)}$ now in $\operatorname{End}(H)$ :

$$
\phi \circ \psi\left(h h^{\prime}\right)=\left(\phi^{(1)} \circ \psi^{(1)}(h)\right) \cdot\left(\phi^{(2)} \circ \psi^{(2)}\left(h^{\prime}\right)\right) .
$$

The last identity in particular holds when $A=H$, that is, in $\operatorname{End}(H)$.

Proof. Indeed, we have:

$$
\begin{aligned}
\phi * \psi\left(h h^{\prime}\right) & =\phi\left(h^{(1)} h^{\prime(1)}\right) \cdot \psi\left(h^{(2)} h^{\prime(2)}\right) \\
& =\phi^{(1)}\left(h^{(1)}\right) \cdot \phi^{(2)}\left(h^{\prime(1)}\right) \cdot \psi^{(1)}\left(h^{(2)}\right) \cdot \psi^{(2)}\left(h^{(2)}\right) \\
& =\phi^{(1)}\left(h^{(1)}\right) \cdot \psi^{(1)}\left(h^{(2)}\right) \cdot \phi^{(2)}\left(h^{\prime(1)}\right) \cdot \psi^{(2)}\left({h^{\prime}}^{(2)}\right) \\
& =\left(\phi^{(1)} * \psi^{(1)}(h)\right) \cdot\left(\phi^{(2)} * \psi^{(2)}\left(h^{\prime}\right)\right),
\end{aligned}
$$

an identity that we also write, for later use,

$$
(\phi * \psi)^{(1)} \otimes(\phi * \psi)^{(2)}=\left(\phi^{(1)} \otimes \phi^{(2)}\right) *\left(\psi^{(1)} \otimes \psi^{(2)}\right) .
$$

We also clearly have:

$$
\phi \circ \psi\left(h h^{\prime}\right)=\phi\left(\psi^{(1)}(h) \psi^{(2)}\left(h^{\prime}\right)\right)=\left(\phi^{(1)} \circ \psi^{(1)}(h)\right) \cdot\left(\phi^{(2)} \circ \psi^{(2)}\left(h^{\prime}\right)\right) .
$$

Corollary 3.1. The graded vector space of infinitesimal characters $\Xi(A)$ is a graded Lie subalgebra of $\operatorname{Lin}(H, A)$ for the Lie bracket induced on the latter by the convolution product.

Proof. Indeed, infinitesimal characters are precisely the elements $\alpha$ of $\operatorname{Lin}(H, A)$ such that:

$$
\alpha^{(1)} \otimes \alpha^{(2)}=\alpha \otimes e+e \otimes \alpha \quad \text { satisfy, for any } h, h^{\prime} \in H: \quad \alpha^{(1)}(h) \cdot \alpha^{(2)}\left(h^{\prime}\right)=\alpha\left(h h^{\prime}\right) .
$$

According to the foregoing lemma, for $\alpha$ and $\beta$ two graded infinitesimal characters, we obtain:

$$
\begin{aligned}
{[\alpha, \beta]\left(h h^{\prime}\right):=} & (\alpha * \beta-\beta * \alpha)\left(h h^{\prime}\right) \\
= & \pi_{A}[(\alpha \otimes e+e \otimes \alpha) *(\beta \otimes e+e \otimes \beta) \\
& -(\beta \otimes e+e \otimes \beta) *(\alpha \otimes e+e \otimes \alpha)]\left(h \otimes h^{\prime}\right) \\
= & {[\alpha, \beta](h) \cdot e\left(h^{\prime}\right)+e(h) \cdot[\alpha, \beta]\left(h^{\prime}\right), }
\end{aligned}
$$

hence the corollary follows. 
Proposition 3.1. The enveloping algebra $U(\Xi(A))$ of the Lie algebra $\Xi(A)$ maps naturally to the convolution subalgebra of $\operatorname{Lin}(H, A)$ generated by $\Xi(A)$.

The existence of that natural algebra map from $U(\Xi(A))$ to $\operatorname{Lin}(H, A)$ follows from the previous lemma and from the universal property of enveloping algebras.

Notice that $U(\Xi(A))$ is also, as the enveloping algebra of a graded Lie algebra, a graded connected cocommutative Hopf algebra, which we call the Hopf algebra $\operatorname{Char}_{H}(A)$, or just $\operatorname{Char}(A)$, of characteristic functions on $H$ (with values in $A$ ). We write $\bar{*}$ for the product on $\operatorname{Char}(A)$ and use $\Delta$ for its coproduct. Thus by definition of $\operatorname{Char}(A)$ the primitive elements are the infinitesimal $A$-valued characters of $H$. Besides providing Hopf algebra tools for the study of Feynman rules, the Hopf algebra of characteristic functions - and the associated pro-unipotent groupwill play a crucial role in Section 10, when relating the FLA approach to renormalization to the Connes-Marcolli motivic Galois group.

Notice that $\Delta$ is not defined in general on $\operatorname{Lin}(H, A)$, and neither on the image of $\operatorname{Char}(A)$ in $\operatorname{Lin}(H, A)$, see [34] and [45] for a discussion on the subject in the particular case $A=H$.

Proposition 3.2. We have, for any $\phi \in \operatorname{Char}(A)$ and any $h, h^{\prime} \in H$, the reciprocity law:

$$
\phi\left(h h^{\prime}\right)=\phi^{(1)}(h) \cdot \phi^{(2)}\left(h^{\prime}\right),
$$

where we use the Sweedler notation for $\Delta(\phi)$, and where the action of $\phi$ on $H$ is induced by the natural map from $\operatorname{Char}(A)$ to $\operatorname{Lin}(H, A)$.

Proof. This is true when $\phi$ is an infinitesimal character. According to the previous proposition, for $\phi, \phi^{\prime}$ in $\operatorname{Char}(A)$, we have: $\phi \bar{*} \phi^{\prime}\left(h h^{\prime}\right)=\phi * \phi^{\prime}\left(h h^{\prime}\right)$. Due to the Lemma 3.1, it follows that the identity holds for $\phi \bar{*} \phi^{\prime}$ if it holds for $\phi$ and $\phi^{\prime}$. Since $\operatorname{Char}(A)$ is generated as an associative algebra by infinitesimal characters, the proposition follows.

We remark that the reciprocity law can be rewritten:

$$
\phi \circ \pi=\pi_{A} \circ \Delta(\phi) .
$$

In the cocommutative case, the identity playing a similar role (mutatis mutandis) is [45]:

$$
\delta \circ \phi=\Delta(\phi) \circ \delta .
$$

As a consequence of Proposition 3.2, the set $G^{\prime}(A)$ of group-like elements in Char $(A)$ maps to characters, that is elements of $G(A)$ - since the identity $\Delta(\phi)=\phi \otimes \phi$ in $\operatorname{Char}(A)$ translates into the identity $\phi\left(h h^{\prime}\right)=\phi(h) \phi\left(h^{\prime}\right)$ in $H$. We show now that, as usual, the convolution exponential and logarithm maps are inverse bijections from $\Xi(A)$ onto $G(A)$ and from $\Xi(A)$ onto $G^{\prime}(A)$. Indeed, for any $\alpha \in \Xi(A)$, we have in $\operatorname{Char}(A)$ :

$$
\begin{aligned}
\Delta(\exp (\alpha)) & =\exp (\Delta(\alpha))=\exp (\alpha \otimes e+e \otimes \alpha)=\exp (\alpha \otimes e) \mp \exp (e \otimes \alpha) \\
& =(\exp (\alpha) \otimes e) *(e \otimes \exp (\alpha))=\exp (\alpha) \otimes \exp (\alpha),
\end{aligned}
$$

which also implies that we have $\exp (\alpha) \in G(A)$ in $\operatorname{Lin}(H, A)$. We have used first that $\alpha$ is a graded infinitesimal character, then that $\alpha \otimes e$ and $e \otimes \alpha$ commute. The other way round, if $\gamma$ is a character:

$$
\begin{aligned}
\log (\gamma)\left(h h^{\prime}\right) & =\pi_{A}\left(\log (\gamma \otimes \gamma)\left(h \otimes h^{\prime}\right)\right) \\
& =\pi_{A}\left((\log (\gamma \otimes e)+\log (e \otimes \gamma))\left(h \otimes h^{\prime}\right)\right) \\
& =\pi_{A}\left((\log (\gamma) \otimes e+e \otimes \log (\gamma))\left(h \otimes h^{\prime}\right)\right) \\
& =\log (\gamma)(h) \cdot e\left(h^{\prime}\right)+e(h) \cdot \log (\gamma)\left(h^{\prime}\right),
\end{aligned}
$$


whereas if $\gamma \in G^{\prime}(A)$ :

$$
\begin{aligned}
\Delta(\log \gamma) & =\log (\Delta \gamma)=\log (\gamma \otimes \gamma) \\
& =\log ((\gamma \otimes e) \bar{*}(e \otimes \gamma))=\log \gamma \otimes e+e \otimes \log \gamma
\end{aligned}
$$

Corollary 3.2. The natural algebra map from $\operatorname{Char}(A)$ to $\operatorname{Lin}(H, A)$ induces a bijection between the group $G^{\prime}(A)$ of group-like elements in $\operatorname{Char}(A)$ and $G(A)$, the group of $A$-valued characters on $H$.

We identify $G(A)$ with $G^{\prime}(A)$ henceforth. In particular, both the identity map $I$ and the antipode $S$ can be viewed as elements of $\operatorname{Char}(H)$, and we won't distinguish between $I, S$ and their respective preimages in $\operatorname{Char}(H)$.

\section{Logarithmic DeRIVATIVES And the Dynkin operator}

Although the logarithm is the simplest bijection from group-like elements to primitive elements in a graded connected cocommutative Hopf algebra, the most relevant bijection in view of applications to renormalization is a bit subtler. It is a kind of logarithmic derivative, closely related to a Lie idempotent known as the Dynkin idempotent. Presently we survey the properties of the Dynkin operator (the composition of the Dynkin idempotent with the grading map $Y)$ pertinent to our forthcoming computations, and also obtain new results on the operator, such as the existence of the advertised bijection between $G(A)$ and $\Xi(A)$. The results generalize the fine properties of Hopf algebras encapsulated in the notion of descent algebra of a Hopf algebra [44]. They rely as well on the Hopf algebraic treatment of the Dynkin operator given in [45], and on more classical Lie theoretic properties of the operator. We give in particular closed formulas for the inverse map to Dynkin's operator, i.e., from $\Xi(A)$ to $G(A)$.

The classical Dynkin operator is defined as follows. Let $X=\left\{x_{1}, \ldots, x_{n}, \ldots\right\}$ be an alphabet. The tensor algebra $T(X):=\bigoplus_{n>0} T_{n}(X)$ over $X$ is a cocommutative graded Hopf algebra with the set of words $x_{i_{1}} \ldots x_{i_{l}}$ as a linear basis in degree $l$. It is also, canonically, the enveloping algebra of the FLA $\operatorname{Lie}(X)$ over $X$. The product in $T(X)$ is induced by concatenation of words, whereas the coproduct is fully characterized by the property that elements of $X$ are primitive in $T(X)$. The Dynkin operator $D: T(X) \rightarrow \operatorname{Lie}(X)$ is given by:

$$
D\left(x_{i_{1}} \ldots x_{i_{n}}\right)=\left[\ldots\left[\left[x_{i_{1}}, x_{i_{2}}\right], x_{i_{3}}\right], \ldots, x_{i_{n}}\right] \text {; }
$$

with $D_{T_{0}(X)}=0$ and $D_{T_{1}(X)}=\mathrm{id}_{X}$. According to an idea essentially due to von Waldenfels, this iterated bracketing operator can be represented in a more abstract way as the convolution product of the antipode $S$ of $T(X)$ with the grading operator $Y$, acting as the multiplication by $n$ on $T_{n}(X)$ :

$$
D=S * Y ; \quad \text { equivalently } \quad I * D=Y .
$$

The most famous property of $D$ is the Dynkin-Specht-Wever theorem, stating that an element $v$ in $T_{n}(X)$ - a linear combination of words of length $n-$ is in $\operatorname{Lie}(X)$ if and only if $D(v)=n v$. In effect, such a $v$ is in the primitive part of the tensor algebra, and:

$$
n v=Y v=\pi(I \otimes D)(1 \otimes v+v \otimes 1)=D(v) .
$$

The converse is also well known. These definitions and properties have been generalized to bialgebras in [45], that we follow mainly here. However, for our purposes we need to give a somewhat detailed account. Indeed, that reference as well as the classical theory of the Dynkin operator do focus on the study of graded connected cocommutative Hopf algebras, whereas we are mainly interested here in commutative Hopf algebras. The interested reader will find 
further historical and technical information about the Dynkin operator and its relevance to Lie computations, as well as other classical Lie idempotents, in references [31] and [47].

So let $H$ be graded, connected and commutative. Since $I \in \operatorname{Char}(H)$, so its graded components $I_{n} \in \operatorname{Char}(H)$. Notice, for further use, that the subalgebra of Char $(H)$ generated by the $I_{n}$ maps to the descent algebra of $H$ - the convolution subalgebra of $\operatorname{End}(H)$ generated by the $I_{n}$, see [44]. Moreover, the grading operator $Y:=\sum_{n \in \mathbb{N}} n I_{n}$ belongs to Char $(H)$. Its coproduct is given by:

$$
\Delta(Y)=Y \otimes I+I \otimes Y
$$

another way of expressing that $Y$ is a derivation of $H$ :

$$
Y\left(h h^{\prime}\right)=Y(h) h^{\prime}+h Y\left(h^{\prime}\right) .
$$

Let us adopt the notation $Y f$ for $f \circ Y$, according to the custom in distribution theory. Under this guise the operator $Y$ extends naturally to a derivation on $\operatorname{Lin}(H, A)$. We find, with $f, g \in \operatorname{Lin}(H, A)$ and $h \in H$ :

$$
\begin{aligned}
Y(f * g)(h) & :=f * g(Y(h))=|h|(f * g)(h) \\
& =|h| f\left(h^{(1)}\right) g\left(h^{(2)}\right) \\
& =\left|h^{(1)}\right| f\left(h^{(1)}\right) g\left(h^{(2)}\right)+\left|h^{(2)}\right| f\left(h^{(1)}\right) g\left(h^{(2)}\right) \\
& =Y f * g(h)+f * Y g(h),
\end{aligned}
$$

where we used that $\Delta(Y(h))=|h| \Delta(h)=\left(\left|h^{(1)}\right|+\left|h^{(2)}\right|\right) h^{(1)} \otimes h^{(2)}$.

Proposition 4.1. Convolution of the $H$-valued character $S$ with any derivation d of $H$ yields an $H$-valued infinitesimal character.

Proof. Indeed, since $d$ is a derivation, we have $d\left(h h^{\prime}\right)=d(h) h^{\prime}+h d\left(h^{\prime}\right)$. Since, furthermore, $\Delta(S)=S \otimes S$, we get:

$$
\begin{aligned}
(S * d)\left(h h^{\prime}\right) & =\pi \circ[(S \otimes S) *(d \otimes I+I \otimes d)]\left(h \otimes h^{\prime}\right) \\
& =\pi \circ[(S * d) \otimes(S * I)+(S * I) \otimes(S * d)]\left(h \otimes h^{\prime}\right) \\
& =S * d(h) \cdot e\left(h^{\prime}\right)+e(h) \cdot S * d\left(h^{\prime}\right),
\end{aligned}
$$

hence the proposition follows.

Corollary 4.1. The Dynkin operator $D:=S * Y$ is an $H$-valued infinitesimal character of $H$.

Notice also that $D$ satisfies $D \circ D=D \circ Y$-in other terms, $D$ is an idempotent up to a scalar factor depending on the grading, that is, $D$ is a quasi-idempotent. Equivalently, $D \circ Y^{-1}$ is an idempotent on $H^{+}$(also known when $H$ is the cotensor algebra $T^{*}(X)$ - see below- as the Dynkin idempotent). Indeed, for any $h \in H$,

$$
D \circ D(h)=D \circ(S * Y)(h)=D\left(S\left(h^{(1)}\right) Y\left(h^{(2)}\right)\right) .
$$

However, since $D$ is an infinitesimal character, $D\left(h h^{\prime}\right)=0$ if $h, h^{\prime} \in H^{+}$and therefore,

$$
D \circ D(h)=D\left(S(h) Y\left(1_{H}\right)+S\left(1_{H}\right) Y(h)\right)=D \circ Y(h)
$$

since $Y\left(1_{H}\right)=0$.

Proposition 4.2. Right composition with an infinitesimal character $\alpha \in \Xi(H)$ induces a map from $G(H)$ to $\Xi(H)$. This also holds for $G(A)$ and $\Xi(A)$, where $A$ is an arbitrary commutative unital algebra. 
Proof. Indeed, let $\gamma \in G(H)$ or $G(A)$, we have:

$$
\gamma \circ \alpha\left(h h^{\prime}\right)=\gamma \circ \alpha(h) e\left(h^{\prime}\right)+e(h) \gamma \circ \alpha\left(h^{\prime}\right),
$$

by virtue of Lemma 3.1, since $\gamma \circ e=e$ for any character $\gamma$.

Corollary 4.2. Right composition with the Dynkin operator $D$ induces a map from $G(A)$ to $\Xi(A)$.

In general, for $\gamma$ belonging to $G(H)$ or $G(A)$ and any $f_{1}, \ldots, f_{k} \in \operatorname{End}(H)$, we have the distributive property:

$$
\gamma \circ\left(f_{1} * \cdots * f_{k}\right)=\left(\gamma \circ f_{1}\right) * \cdots *\left(\gamma \circ f_{k}\right)
$$

Particularly,

$$
\gamma \circ D=\gamma \circ(S * Y)=\gamma^{-1} * Y \gamma
$$

Theorem 4.1. Right composition with $D$ is a bijective map from $G(H)$ to $\Xi(H)$. The inverse map is given by:

$$
\Gamma: \alpha \in \Xi(H) \longmapsto \sum_{n} \sum_{\begin{array}{c}
k_{1}, \ldots, k_{l} \in \mathbb{N}^{*} \\
k_{1}+\cdots+k_{l}=n
\end{array}} \frac{\alpha_{k_{1}} * \cdots * \alpha_{k_{l}}}{k_{1}\left(k_{1}+k_{2}\right) \ldots\left(k_{1}+\cdots+k_{l}\right)} \in G(H) .
$$

The theorem also holds if $G(H)$ and $\Xi(H)$ are replaced by $G(A)$, respectively $\Xi(A)$.

We show first that $\Gamma$ is a left inverse to right composition with $D$. The following lemma has been obtained in [31] in the setting of noncommutative symmetric functions and quasideterminants. We include the proof, which is elementary.

Lemma 4.1. For $n \geq 1$ we have:

$$
I_{n}=\sum_{\substack{k_{1}, \ldots, k_{l} \in \mathbb{N}^{*} \\ k_{1}+\cdots+k_{l}=n}} \frac{D_{k_{1}} * \cdots * D_{k_{l}}}{k_{1}\left(k_{1}+k_{2}\right) \cdots\left(k_{1}+\cdots+k_{l}\right)} .
$$

Proof. As already remarked, the definition of $D$ implies $I * D=I * S * Y=Y$. In particular, since $D_{0}=0$ :

$$
Y_{n}=n I_{n}=(I * D)_{n}=\sum_{i=1}^{n} I_{n-i} * D_{i} .
$$

Inserting recursively the value of $I_{i}$ in the right member of the identity, we obtain:

$$
\begin{aligned}
I_{n} & =\frac{D_{n}}{n}+\sum_{i=1}^{n-1} \sum_{1 \leq j \leq n-i} \frac{I_{n-i-j} * D_{j} * D_{i}}{(n-i) n} \\
& =\frac{D_{n}}{n}+\sum_{\substack{j+i=n \\
i, j \neq 0}} \frac{D_{j} * D_{i}}{j \cdot n}+\sum_{i=1}^{n-1} \sum_{1 \leq j \leq n-i-1} \frac{I_{n-i-j} * D_{j} * D_{i}}{(n-i) n} \\
& =\sum_{\substack{k_{1}, \ldots, k_{l} \in \mathbb{N}^{*} \\
k_{1}+\cdots+k_{l}=n}} \frac{D_{k_{1}} * \cdots * D_{k_{l}}}{k_{1}\left(k_{1}+k_{2}\right) \cdots\left(k_{1}+\cdots+k_{l}\right)}
\end{aligned}
$$


Now we compute $\gamma=\gamma \circ I$, where $I$ is expanded according to the previous lemma, yielding:

$$
\begin{aligned}
\gamma & =e+\gamma \circ\left\{\sum_{n \in \mathbb{N}^{*}} \sum_{\substack{k_{1}, \ldots, k_{l} \in \mathbb{N}^{*} \\
k_{1}+\cdots+k_{l}=n}} \frac{D_{k_{1}} * \cdots * D_{k_{l}}}{k_{1}\left(k_{1}+k_{2}\right) \ldots\left(k_{1}+\cdots+k_{l}\right)}\right\} \\
& =e+\sum_{n \in \mathbb{N}^{*}} \sum_{\substack{k_{1}, \ldots, k_{l} \in \mathbb{N}^{*} \\
k_{1}+\cdots+k_{l}=n}} \frac{\gamma \circ D_{k_{1}} * \cdots * \gamma \circ D_{k_{l}}}{k_{1}\left(k_{1}+k_{2}\right) \ldots\left(k_{1}+\cdots+k_{l}\right)} .
\end{aligned}
$$

As $D$ preserves the grading, it follows that $\Gamma$ is a left inverse to the right composition with $D$.

Similar calculations help to prove that $\Gamma$ is character-valued, that is, is actually a map from $\Xi(H)$ to $G(H)$. Indeed, let $\alpha$ be any infinitesimal character in $\Xi(H)$. Then we have in $\operatorname{Char}(H)$ :

$$
\begin{aligned}
& \Delta(\Gamma(\alpha))=e \otimes e+\sum_{n>0} \sum_{\substack{k_{1}, \ldots, k_{l} \in \mathbb{N}^{*} \\
k_{1}+\cdots+k_{l}=n}} \frac{\Delta\left(\alpha_{k_{1}} \bar{*} \cdots \bar{*} \alpha_{k_{l}}\right)}{k_{1}\left(k_{1}+k_{2}\right) \ldots\left(k_{1}+\cdots+k_{l}\right)} \\
& =e \otimes e+\sum_{n>0} \sum_{\substack{k_{1}, \ldots, k_{l} \in \mathbb{N}^{*} \\
k_{1}+\cdots+k_{l}=n}} \sum_{\substack{I \sqcup J=\left\{k_{1}, \ldots, k_{l}\right\} \\
|I|=m,|J|=p}} \frac{\left(\alpha_{i_{1}} \bar{*} \cdots \bar{*} \alpha_{i_{m}}\right) \otimes\left(\alpha_{j_{1}} * \cdots \bar{*} \alpha_{j_{p}}\right)}{k_{1}\left(k_{1}+k_{2}\right) \ldots\left(k_{1}+\cdots+k_{l}\right)},
\end{aligned}
$$

where $I=\left\{i_{1}, \ldots, i_{m}\right\}, J=\left\{j_{1}, \ldots, j_{p}\right\}$ and we have used that the $\alpha_{i_{m}}$ are all infinitesimal characters. Particularly, the assertion we intend to prove, that is $\Delta(\Gamma(\alpha))=\Gamma(\alpha) \otimes \Gamma(\alpha)$, amounts to the equality:

$$
\begin{aligned}
& \left(e+\sum_{n>0} \sum_{\substack{k_{1}, \ldots, k_{l} \in \mathbb{N}^{*} \\
k_{1}+\cdots+k_{l}=n}} \frac{\alpha_{k_{1}} \bar{*} \cdots \bar{*} \alpha_{k_{l}}}{k_{1}\left(k_{1}+k_{2}\right) \ldots\left(k_{1}+\cdots+k_{l}\right)}\right)^{\otimes 2} \\
& =e \otimes e+\sum_{n>0} \sum_{\substack{k_{1}, \ldots, k_{l} \in \mathbb{N}^{*} \\
k_{1}+\cdots+k_{l}=n}} \sum_{\substack{I \sqcup J=\left\{k_{1}, \ldots, k_{l}\right\} \\
|I|=m,|J|=p}} \frac{\left(\alpha_{i_{1}} \bar{*} \cdots \bar{*} \alpha_{i_{m}}\right) \otimes\left(\alpha_{j_{1}} \bar{*} \cdots \bar{*} \alpha_{j_{p}}\right)}{k_{1}\left(k_{1}+k_{2}\right) \ldots\left(k_{1}+\cdots+k_{l}\right)} .
\end{aligned}
$$

This follows from the identity:

$$
\begin{aligned}
& \sum_{I \sqcup J=K} \frac{1}{k_{1}\left(k_{1}+k_{2}\right) \ldots\left(k_{1}+\cdots+k_{p+m}\right)} \\
& \quad=\sum_{\substack{i_{1}, \ldots, i_{m} \in \mathbb{N}^{*} \\
j_{1}, \ldots, j_{p} \in \mathbb{N}^{*}}} \frac{1}{i_{1}\left(i_{1}+i_{2}\right) \ldots\left(i_{1}+\cdots+i_{m}\right)} \cdot \frac{1}{j_{1}\left(j_{1}+j_{2}\right) \ldots\left(j_{1}+\cdots+j_{p}\right)},
\end{aligned}
$$

where $K$ runs over all sequences $\left(k_{1}, \ldots, k_{p+l}\right)$ obtained by shuffling the sequences $I$ and $J$. In turn, the identity follows if the equation $\Delta(\Gamma(\alpha))=\Gamma(\alpha) \otimes \Gamma(\alpha)$ - that is, $\Gamma(\alpha) \in G(H)$ holds for a particular choice of $H$ and $\alpha$ such that the $\alpha_{i}$ form a family of algebraically independent generators of a subalgebra of the convolution algebra $\operatorname{End}(H)$-which are therefore also algebraically independent as elements of Char $(H)$.

So, let us consider the particular case $H=T^{*}(X)$ where $H$ is the graded dual of the enveloping algebra of the FLA over an infinite alphabet $X$ and $\alpha=D$ is the Dynkin operator. Then, we already know that $\Gamma(D)=I$, due to the previous lemma, so that $\Gamma(D)$ is grouplike in $\operatorname{Char}\left(T^{*}(X)\right)$. On the other hand, as is well known, the graded components of the Dynkin operator are algebraically independent in the convolution algebra $\operatorname{End}\left(T^{*}(X)\right)$, and 
the identity follows. (For details on the algebraic independence of the graded components of the Dynkin operator, consult $[31,47]$.)

Although we have preferred to give a conceptual proof based on FLAs, let us mention that identity (2) is elementary, and well known in boson Fock space theory. It also follows e.g. from the shuffle identity for the product of two iterated integrals [48]:

$$
\begin{aligned}
& \sum_{I \sqcup J=K} \int_{0}^{1} x_{p+l}^{k_{p+m}-1} \ldots \int_{0}^{x_{2}} x_{1}^{k_{1}-1} d x_{1} \ldots d x_{p+m} \\
& \quad=\int_{0}^{1} x_{m}^{i_{m}-1} \ldots \int_{0}^{x_{2}} x_{1}^{i_{1}-1} d x_{1} \ldots d x_{m} \cdot \int_{0}^{1} y_{p}^{j_{p}-1} \ldots \int_{0}^{y_{2}} y_{1}^{j_{1}-1} d y_{1} \ldots d y_{p},
\end{aligned}
$$

which generalizes the integration by parts rule.

To conclude the proof of Theorem 4.1 we show that $\Gamma$ is also a right inverse to the composition with $D$. We contend that, for any $h$ in the augmentation ideal of $H$ and arbitrary $\alpha \in \Xi(H)$, the following relation holds:

$$
\alpha(h)=\Gamma(\alpha)^{-1} * Y \Gamma(\alpha)(h) \quad \text { or, equivalently, } \quad Y \Gamma(\alpha)(h)=\Gamma(\alpha) * \alpha(h) .
$$

Indeed, we have:

$$
\begin{aligned}
Y \Gamma(\alpha)(h) & :=|h| \sum_{\substack{k_{1}, \ldots, k_{l} \in \mathbb{N}^{*} \\
k_{1}+\cdots+k_{l}=|h|}} \frac{\alpha_{k_{1}} * \cdots * \alpha_{k_{l}}}{k_{1}\left(k_{1}+k_{2}\right) \cdots\left(k_{1}+\cdots+k_{l}\right)}(h) \\
& =\sum_{\substack{k_{1}, \ldots, k_{l} \in \mathbb{N}^{*} \\
k_{1}+\cdots+k_{l}=|h|}} \frac{\alpha_{k_{1}} * \cdots * \alpha_{k_{l-1}}}{k_{1}\left(k_{1}+k_{2}\right) \cdots\left(k_{1}+\cdots+k_{l-1}\right)} * \alpha_{k_{l}}(h) \\
& =\Gamma(\alpha) * \alpha(h) .
\end{aligned}
$$

This together with the fact that $\Gamma(\alpha) \in G(H)$ for $\alpha \in \Xi(H)$ implies:

$$
\Gamma(\alpha) \circ D=\Gamma(\alpha)^{-1} * Y \Gamma(\alpha)=\alpha .
$$

Our task is over.

When $H$ is both commutative and cocommutative the convolution product is commutative and $\gamma \circ D=Y \log \gamma:=\log (\gamma) \circ Y$. In particular in this case $D=Y \log I$. This was put to good use in [7]. Thus clearly $D$, in the general case, is a noncommutative logarithmic derivative; and the inverse Dynkin operator $\Gamma$ a extremely powerful tool. We finally remark that $Y * S$, corresponding, in the free cocommutative case and as an operator from the tensor algebra to the FLA, to the right-to-left iteration of bracketings, is another possible form for the noncommutative logarithmic derivative, leading in particular to $\gamma \circ(Y * S)=Y \gamma * \gamma^{-1}$. More generally, any interpolation of the form $S^{a} * Y * S^{b}$, with $a+b=1$, yields a notion of noncommutative logarithmic derivative.

\section{Algebraic BWH decomposition of Characters}

In this section we summarize previous research on Rota-Baxter operators, relevant for our purpose. Let $H$ be still graded, connected and commutative and let again $A$ stand for a commutative unital algebra. Assume the algebra $A$ to split directly, $A=A_{+} \oplus A_{-}$, into the subalgebras $A_{+}, A_{-}$with $1_{A} \in A_{+}$. We denote the projectors to $A_{ \pm}$by $R_{ \pm}$, respectively. 
The pair $\left(A, R_{-}\right)$is a special case of a (weight one) Rota-Baxter algebra [23] since $R_{-}$, and similarly $R_{+}$, satisfies the relation:

$$
R_{-}(x) \cdot R_{-}(y)+R_{-}(x \cdot y)=R_{-}\left(R_{-}(x) \cdot y+x \cdot R_{-}(y)\right), \quad x, y \in A .
$$

The reader may verify that integration by parts rule is just the weight zero Rota-Baxter identity, that is, the second term on left hand side of (3) is absent. One easily shows that $\operatorname{Lin}(H, A)$ with the idempotent operator $\mathcal{R}_{-}$defined by $\mathcal{R}_{-}(f)=R_{-} \circ f$, for $f \in \operatorname{Lin}(H, A)$, is a (in general not commutative) unital Rota-Baxter algebra [23].

The subspace $L^{(1)}$ of $\operatorname{Lin}(H, A)$ made of linear maps that send the Hopf algebra unit to zero forms a Lie algebra with $\Xi(A) \subset L^{(1)}$ as a Lie subalgebra. To $L^{(1)}$ does correspond the group $G_{0}=e+L^{(1)}=\exp \left(L^{(1)}\right)$ of linear maps sending the Hopf algebra unit to the algebra unit. It contains the group of characters $G(A)$ as the subgroup $\exp (\Xi(A))$. Due to the characterization of infinitesimal characters as maps that vanish on the square of the augmentation ideal of $H$, both $\mathcal{R}_{+}(\Xi(A))$ and $\mathcal{R}_{-}(\Xi(A))$ embed into $\Xi(A)$. In particular, both are Lie subalgebras of $\Xi(A)$.

The Lie algebra decomposition, $\Xi(A)=\mathcal{R}_{+}(\Xi(A)) \oplus \mathcal{R}_{-}(\Xi(A))$ lifts to the group of characters $G(A)$ as follows. Recall the Baker-Campbell-Hausdorff (BCH) formula [47] for the product of two exponentials, that we write:

$$
\exp (x) \exp (y)=\exp (x+y+\operatorname{BCH}(x, y))
$$

In $[23,24]$ the following non-linear map was defined, whose properties where further explored in [27]. See also [42]. For $f \in L^{(1)}$, define $\chi^{\mathcal{R}_{-}}(f)=\lim _{n \rightarrow \infty} \chi_{n}^{\mathcal{R}_{-}}(f)$ where $\chi_{n}^{\mathcal{R}_{-}}(f)$ is given by what we call the $\mathrm{BCH}$ recursion:

$$
\begin{aligned}
\chi_{0}^{\mathcal{R}_{-}}(f) & =f, \quad \chi_{1}^{\mathcal{R}_{-}}(f)=f-\mathrm{BCH}\left(\mathcal{R}_{-}(f), \mathcal{R}_{+}(f)\right), \ldots, \\
\chi_{n+1}^{\mathcal{R}_{-}}(f) & =f-\mathrm{BCH}\left(\mathcal{R}_{-}\left(\chi_{n}^{\mathcal{R}_{-}}(f)\right), \mathcal{R}_{+}\left(\chi_{n}^{\mathcal{R}_{-}}(f)\right)\right) .
\end{aligned}
$$

Then the fixed-point map $\chi^{\mathcal{R}_{-}}: L^{(1)} \rightarrow L^{(1)}$ satisfies:

$$
\chi^{\mathcal{R}_{-}}(f)=f-\operatorname{BCH}\left(\mathcal{R}_{-}\left(\chi^{\mathcal{R}_{-}}(f)\right), \mathcal{R}_{+}\left(\chi^{\mathcal{R}_{-}}(f)\right)\right) .
$$

The superscript $\mathcal{R}_{-}$is justified by the dependency of the limit on the Rota-Baxter operator, and the following result.

Lemma 5.1. The map $\chi^{\mathcal{R}_{-}}$in (4) solves the simpler recursion:

$$
\chi^{\mathcal{R}_{-}}(f)=f+\mathrm{BCH}\left(-\mathcal{R}_{-}\left(\chi^{\mathcal{R}_{-}}(f)\right), f\right), \quad f \in L^{(1)} .
$$

Following [27], the following factorization theorem holds.

Theorem 5.1. For any $f \in L^{(1)}$, we have the unique factorization:

$$
\exp (f)=\exp \left(\mathcal{R}_{-}\left(\chi^{\mathcal{R}_{-}}(f)\right)\right) * \exp \left(\mathcal{R}_{+}\left(\chi^{\mathcal{R}_{-}}(f)\right)\right)
$$

Uniqueness of the factorization follows from $R_{-}$being idempotent. In the particular case that $f \in \Xi(A)$, the $\mathrm{BCH}$ recursion (4) takes place inside the Lie algebra $\Xi(A)$, and the decomposition of $\exp (f)$ holds therefore inside the group of characters $G(A)$. In particular, it follows ultimately from Theorem 5.1 that $G(A)$ decomposes as a set as the product of two subgroups:

$$
G(A)=G_{-}(A) * G_{+}(A), \quad \text { where } \quad G_{-}(A)=\exp \left(\mathcal{R}_{-}(\Xi(A))\right), G_{+}(A)=\exp \left(\mathcal{R}_{+}(\Xi(A))\right) .
$$


Corollary 5.1. For any $\gamma=\exp (\alpha) \in G(A)$, with $\alpha \in \Xi(A)$, we have unique $\alpha_{ \pm}:=$ $\mathcal{R}_{ \pm}\left(\chi^{\mathcal{R}_{-}}(\alpha)\right) \in \mathcal{R}_{ \pm}(\Xi(A))$, and unique characters $\gamma_{ \pm}:=\exp \left( \pm \alpha_{ \pm}\right) \in G_{ \pm}(A)$ such that:

$$
\gamma=\gamma_{-}^{-1} * \gamma_{+} \text {. }
$$

A remark is in order. The factorization in Theorem 5.1 is true for any (filtration preserving) linear map $P$ on $\operatorname{Lin}(H, A)$, that is, for $\chi^{P}(f)$, see [27]. Uniqueness follows from $P$ being idempotent. The Rota-Baxter property (3) implies that both, $G_{ \pm}(A)$ are subgroups. We may reformulate the last statement about the nature of $G_{ \pm}(A)$ in the next lemma, saying that the BWH decomposition of Connes and Kreimer, originally found by a more geometrical method [18], is recovered from Theorem [5.1 by using the Rota-Baxter relation [23, 26, 27]:

Lemma 5.2. For any $\gamma=\exp (\alpha)$ the unique characters $\gamma_{ \pm}:=\exp \left( \pm \alpha_{ \pm}\right) \in G_{ \pm}(A)$ in the previous corollary solve the equations:

$$
\gamma_{ \pm}=e \pm \mathcal{R}_{ \pm}\left(\gamma_{-} *(\gamma-e)\right)
$$

Proof. There is a sharpened version [24] of Atkinson's theorem [1], stating that the characters $\gamma, \gamma_{ \pm}$of (5) verify $\gamma_{-}=e-\mathcal{R}_{-}\left(\gamma_{-} *(\gamma-e)\right)$ and $\gamma_{+}=e-\mathcal{R}_{+}\left(\gamma_{+} *\left(\gamma^{-1}-e\right)\right)$. Now:

$$
\gamma_{+} *\left(\gamma^{-1}-e\right)=\gamma_{-} * \gamma *\left(\gamma^{-1}-e\right)=\gamma_{-} *(e-\gamma)
$$

gives (6).

\section{ON RENORMALIZATION PROCEDURES}

Prima facie in pQFT, to a Feynman graph $F$ does correspond by the Feynman rules a multiple $d$-dimensional momentum space integral. Each independent loop in a diagram yields one integration:

$$
F \mapsto J_{F}(p)=\left[\int \prod_{l=1}^{|F|} d^{d} k_{l}\right] I_{F}(p, k) .
$$

Here $|F|$ is the number of loops, $k=\left(k_{1}, \ldots, k_{|F|}\right)$ are the $|F|$ independent internal (loop) momenta and $p=\left(p_{1}, \ldots, p_{N}\right)$, with $\sum_{k=1}^{N} p_{k}=0$, denote the $N$ external momenta. In the most relevant kind ( $d=4$, renormalizable with dimensionless couplings) of field theories, these integrals suffer from ultraviolet (UV) divergencies. Concretely, under a scale transformation, the integrand behaves as

$$
\left[\prod_{l=1}^{|F|} d^{d}\left(\lambda k_{l}\right)\right] I_{F}(\lambda p, \lambda k) \sim \lambda^{s(F)},
$$

with $s(F)$ the superficial UV degree of divergence of the graph $F$. Power-counting renormalizable theories are such that all interaction terms in the Lagrangian are of dimension $\leq d$; then $s(F)$ is bounded by a number independent of the order of the graph. For instance in the $\varphi_{4}^{4}$ model the superficial UV degree of divergence of a graph with $N$ external legs is:

$$
s(F)=4-N .
$$

The Weinberg-Zimmermann theorem says: "provided all free propagators have nonzero masses, the integral associated to the Feynman graph $F$ is absolutely convergent if its superficial UV degree of divergence and that of each of its one-particle irreducible (1PI) subgraphs is strictly negative". The BPHZ momentum space subtraction method is rooted in this assertion: the idea is to redefine the integrand $I_{F}(p, k)$ of a divergent integral by subtracting from it the first terms of its Taylor expansion in the external momenta $p$, after these subtractions have been performed on the integrands corresponding to the 1PI subgraphs of $F$ that are renormalization 
parts; in such a way the UV degrees of the integral and its subintegrals are lowered until they become all negative. The combinatorics of those subgraph subtractions leads to Bogoliubov's recursive $\bar{R}$-operation and Zimmermann's forest formula; we assume the reader acquainted with the former at least $[13,15,49,52]$.

Less straightforward conceptually, but way more practical, is the DR method [51]. This belongs to the class of regularization prescriptions, which parameterize the divergencies appearing in $J_{F}$ upon introducing non-physical parameters, thereby rendering them formally finite. In $\mathrm{DR}$ one introduces a complex parameter $\varepsilon \in \mathbb{C}$ by changing the integral measure, that is, the space-time dimension, to $\mathrm{D} \in \mathbb{C}$ :

$$
d^{d} k \stackrel{\operatorname{dim} \mathrm{reg}}{\longrightarrow} \mu^{\varepsilon} d^{\mathrm{D}} k,
$$

where $\varepsilon=(d-\mathrm{D})$. Henceforth always $d=4$. The arbitrary parameter $\mu \neq 0$ ('t Hooft's 'unit-mass' parameter) is introduced for dimensional reasons. Take the $\varphi_{4}^{4}$ model: if we wrote the interaction term simply in the form $g \varphi^{4} / 4$ !, then the (naive) dimension of $g$ would be $[g]=\mu^{\varepsilon}$. The redefinition $\tilde{g} \mu^{\varepsilon} \varphi^{4} / 4$ ! of the original vertex in the Lagrangian includes the mass parameter $\mu$, introduced to make $\tilde{g}$ dimensionless. Now, in any given theory there is a rigid relation between the numbers of loops and vertices, for each given $N$-point function. For instance in the $\varphi_{4}^{4}$ model, for graphs associated to the 2-point function the number of vertices is just equal to the number of loops. For graphs associated to the 4-point function the number of vertices is equal to the number of loops plus one, and so an extra $\tilde{g} \mu^{\varepsilon}$ factor comes in; but, because we are correcting the vertex, this extra factor becomes the coupling constant, and is not expanded in powers of the regularization parameter $\varepsilon$; only the expression multiplying it contributes to the renormalization constant $Z_{g}$-with a pole term independent of the mass scale $\mu$. The outcome is that in practice one computes as many $\mu^{\varepsilon}$ factors as loops:

$$
F \longrightarrow J_{F}^{(\varepsilon, \mu)}(p)=\mu^{|F| \varepsilon}\left[\int \prod_{l=1}^{|F|} d^{\mathrm{D}} k_{l}\right] I_{F}(p, k) .
$$

See the discussions in $[18,19]$ and $[35$, Sections 7 and 8] as well. The point is important from the algebraic viewpoint, since it makes the grading of Feynman graphs by the number of loops immediately relevant to the renormalization process.

The next step in DR consists of a specific subtraction rule of those $\varepsilon$-parameterized expressions which allows to take the limit $\varepsilon \downarrow 0$. Now, Connes and Kreimer's BWH decomposition of Feynman rules [18] is extraordinarily well adapted to DR in pQFT. In the Connes-Kreimer paradigm, any renormalizable quantum field theory gives rise to a Hopf algebra $H$ of Feynman graphs, polynomially generated by 1PI Feynman graphs and graded by graph loop number. The coproduct operation of $H$ mirrors the combinatorics of the subgraphs. Looking back to (7), the unrenormalized Feynman integral does define a character because:

$$
I_{F_{1} \cup F_{2}}\left(p_{1}, p_{2}, k_{1}, k_{2}\right)=I_{F_{1}}\left(p_{1}, k_{1}\right) I_{F_{2}}\left(p_{2}, k_{2}\right)
$$

for disjoint graphs $F_{1}, F_{2}$. On the Hopf algebra $H$ the Feynman rules single out a distinguished character $\gamma$ with values in a suitable target algebra of regularized amplitudes. Precisely, Connes and Kreimer establish the above decomposition $G(A)=G_{-}(A) * G_{+}(A)$, for $A$ the algebra of amplitude-valued Laurent series in the dimension defect $\varepsilon$, using the MS scheme in DR on momentum space. The characters $\gamma_{ \pm}$in the decomposition (5) solve Bogoliubov's renormalization formulae - see Corollary 6.1 below - and may be called the renormalized and counterterm character, respectively. The sought after result is given by the 'positive' part in (5). To wit, $\gamma_{+}^{(\varepsilon, \mu)}=\gamma_{-}^{(\varepsilon, \mu)} * \gamma^{(\varepsilon, \mu)}$, and the limit $\gamma_{+}^{(\varepsilon \downarrow 0, \mu)}$ exists, giving the renormalized 
Feynman amplitude. In what follows, when dealing with dimensionally regularized characters we drop the superscript $\varepsilon$. We also forget about the other involved variables, that do not enter our considerations, and just write $\mathbb{C}\left[\left[\varepsilon, \varepsilon^{-1}\right]\right.$ for $A$. Thus $R_{-}$will be the projection onto the subalgebra $A_{-}:=\varepsilon^{-1} \mathbb{C}\left[\varepsilon^{-1}\right]$. In summary, $A$ encodes the Feynman rules within the chosen regularization procedure, whereas the splitting of $A$, hence the projector $R_{-}$, reflects a renormalization scheme within that choice.

Corollary 6.1. The map $\bar{\gamma}:=\gamma_{-} *(\gamma-e)=\gamma_{+}-\gamma_{-}$in (6) gives Bogoliubov's preparation map $\bar{R}$.

Indeed with the Connes-Kreimer definition of the Hopf algebra of Feynman graphs, equations (6) coincide with Bogoliubov's recursive formulas for the counterterm and renormalized parts.

Recalling the remark after Corollary 5.1 we may characterize the Rota-Baxter structure on $A$ as follows. Theorem 5.1 implies that regularized, i.e. $A$-valued, Feynman rules on $H$ factorize uniquely upon the choice of an idempotent linear map on $A$ respectively $\operatorname{Lin}(H, A)$. The extra demand for a Rota-Baxter structure on $A$, and hence on $\operatorname{Lin}(H, A)$ essentially implies the particular recursive nature of the process of perturbative renormalization as encoded in Bogoliubov's preparation map $\bar{R}$ respectively the equations (6).

\section{Locality and the Connes-Kreimer Beta FunCtion}

The results in Sections 3 to 5 apply to any graded connected commutative bialgebra $H$ and any commutative unital algebra $A$ with a direct splitting. In this section we restrict our consideration to the class of Hopf algebra characters possessing a locality property, with $H$ as before. This will correspond to the example given by the Feynman rules for a renormalizable pQFT in DR, using the framework of the MS and the $\overline{\mathrm{MS}}$ [15, Section 5.11.2] schemes. There locality comes from the dependency of DR on the arbitrary 'mass parameter' $\mu$. It is handy to provisionally fix the value of $\mu$ at some convenient reference point $\mu_{0}$. The difference between both schemes boils down to:

$$
\mu_{0}=\bar{\mu}_{0} \frac{e^{\gamma_{E} / 2}}{2 \sqrt{\pi}}
$$

with $\mu_{0}, \bar{\mu}_{0}$ respectively the MS, $\overline{\mathrm{MS}}$ values and $\gamma_{E}$ the Euler-Mascheroni constant. Our aim is to recover by our methods the results in [19,21]; the latter constitute a stronger, algebraic version of a famous theorem by 't Hooft [51]. Place of pride corresponds to the ConnesKreimer abstract beta function. This is a conceptually very powerful beast, giving rise to the ordinary beta function through the (tangent morphism to) the morphism (of unipotent groups) from $G(\mathbb{C})$ to the group of transformations of the coupling constants [19].

Any $f \in \operatorname{Lin}(H, A)$ is now of the form:

$$
f(h)=\sum_{k=-U}^{\infty} f_{: k}(h) \varepsilon^{k}=: f(h ; \varepsilon)
$$

for $h \in H$. Here every $f_{: k} \in \operatorname{Lin}(H, \mathbb{C})$, the dual of $H$; and the last notation is used when we want to emphasize the dependency on the DR parameter $\varepsilon$. If $h$ is a $|h|$-loop 1PI Feynman graph, a general theorem [49] in DR says that $U=|h|$ at non-exceptional momenta.

We define on the group of $A$-valued characters $G(A)$ a one-parameter action of $\mathbb{C}^{*} \ni t$ given for $h$ homogeneous in $H$ by:

$$
\psi^{t}(h ; \varepsilon):=t^{\varepsilon|h|} \psi(h ; \varepsilon) .
$$


Physically this amounts to neatly replacing the $\mu_{0}^{\varepsilon|h|}$ factor present in each dimensionally regularized Feynman diagram (8) by $\left(\mu_{0} t\right)^{\varepsilon|h|}$; that is, the mass scale is changed from $\mu_{0}$ to $t \mu_{0}$ - or from $\bar{\mu}_{0}$ to $t \bar{\mu}_{0}$, as the case might be. As $\varepsilon$ is a complex variable, there is no harm in taking $t$ complex, too.

It is clear that $\psi^{t}$ in (10) is still a character, and that $\left(\psi_{1} * \psi_{2}\right)^{t}=\psi_{1}^{t} * \psi_{2}^{t}$. This last property also holds if $\psi_{1}, \psi_{2}$ in (10) belong more generally to $\operatorname{Lin}(H, A)$. Besides, for future use we store:

$$
t \frac{\partial}{\partial t} \psi^{t}=\varepsilon|h| \psi^{t}(h ; \varepsilon)=\varepsilon Y \psi^{t} \quad \text { such that }\left.\quad t \frac{\partial}{\partial t}\right|_{t=1} \psi^{t}=\varepsilon Y \psi .
$$

For any $t$ and any homogeneous $h \in H$ we have $t^{\varepsilon|h|} \in \mathcal{R}_{+}(A)=A_{+}:=\mathbb{C}[[\varepsilon]]$, so that the one-parameter action on $G(A)$ restricts to a one-parameter action on the group $G_{+}(A)$ :

$$
\psi \in G_{+}(A) \mapsto \psi^{t} \in G_{+}(A) .
$$

We now have for the regularized, but unrenormalized character $\gamma^{t} \in G(A)$ a BWH decomposition:

$$
\gamma^{t}=\left(\gamma^{t}\right)_{-}^{-1} *\left(\gamma^{t}\right)_{+} \cdot
$$

Notice that we write instead $\left(\gamma_{-}\right)^{t}$ and $\left(\gamma_{+}\right)^{t}$ for the image of $\gamma_{-}$and $\gamma_{+}$under the oneparameter group action. The locality property simply states that for the counterterm the following holds.

Theorem 7.1. Let $\gamma$ be a dimensionally regularized Feynman rule character. The counterterm character in the BWH decomposition $\gamma^{t}=\left(\gamma^{t}\right)_{-}^{-1} *\left(\gamma^{t}\right)_{+}$satisfies:

$$
t \frac{\partial\left(\gamma^{t}\right)_{-}}{\partial t}=0 \quad \text { or }\left(\gamma^{t}\right)_{-} \text {is equal to } \gamma_{-} \text {, i.e. independent of } t \text {. }
$$

We say the A-valued characters $\gamma \in G(A)$ with this property are local characters.

The physical reason for this is that the counterterms can be taken mass-independent; this goes back at least to [14]. For this fact, and more details on the physical significance of the locality property in pQFT, we refer the reader to $[3,15,18,21]$.

In the sequel, albeit mustering Dynkin technology, we partly follow the plan in reference [42]. Denote by $G^{\text {loc }}(A)$ the subset of local elements of $G(A)$ and $G_{-}^{\text {loc }}(A)$ the subset of elements in $G^{\text {loc }}(A)$ for which $\psi(h) \in A_{-}$when $h$ has no scalar part.

Proposition 7.1. The set $G^{\mathrm{loc}}(A)$ decomposes into the product $G_{-}^{\mathrm{loc}}(A) * G_{+}(A)$.

Proof. Notice first that $G_{+}(A)$ embeds in $G^{\text {loc }}(A)$, since $\psi^{t} \in G_{+}(A)$ for any $\psi \in G_{+}(A)$. Next, if $\phi$ is local and $\rho \in G_{+}(A)$, then $\phi * \rho$ is local. Indeed, we have:

$$
\phi^{t} * \rho^{t}=\left(\phi^{t}\right)_{-}^{-1} *\left(\phi^{t}\right)_{+} * \rho^{t},
$$

with polar part the one of $\phi^{t}$, which is constant since $\phi$ is local. Let $\phi$ still be local. Then $\phi * \phi_{+}^{-1}=\phi_{-}^{-1}$, and the proposition follows if we can show $\phi_{-}^{-1} \in G_{-}^{\text {loc }}(A)$. Now, if $\phi$ is local, we have:

$$
\left(\phi_{-}^{-1}\right)^{t} *\left(\phi_{+}\right)^{t}=\phi^{t}=\left(\phi^{t}\right)_{-}^{-1} *\left(\phi^{t}\right)_{+} ;
$$

so that the BWH decomposition of $\left(\phi_{-}^{-1}\right)^{t}$ is given by:

$$
\left(\phi_{-}^{-1}\right)^{t}=\left(\phi^{t}\right)_{-}^{-1} *\left(\phi^{t}\right)_{+} *\left(\left(\phi_{+}\right)^{t}\right)^{-1},
$$

with polar part $\left(\phi^{t}\right)_{-}$, the one of $\phi$, constant and equal to $\phi_{-}$. 
Now we wish to complete the study of locality by understanding how the decomposition of $G^{\text {loc }}(A)$, which is a refinement of the decomposition $G(A)=G_{-}(A) * G_{+}(A)$, is reflected at the Lie algebra level. More precisely, we would like to know if there is a subspace of $\mathcal{R}_{-}(\Xi(A))$ naturally associated to $G_{-}^{\text {loc }}(A)$. The answer (quite surprising at first sight) is simple and well known to physicists: beta functions are enough. As shown below, an excellent tool to understand this is the Dynkin operator pair. Let now $\beta \in \Xi(\mathbb{C})$ be a scalar-valued infinitesimal character. Notice that $\beta / \varepsilon$ can be regarded as an element of $\mathcal{R}_{-}(\Xi(A))$.

Proposition 7.2. With $\Gamma$ as defined in Eq. (11) of Theorem 4.1, we find:

$$
\psi_{\beta}:=\Gamma(\beta / \varepsilon) \in G_{-}^{\mathrm{loc}}(A) .
$$

Proof. From Eq. (11) it is clear that :

$$
\psi_{\beta}=\Gamma(\beta / \varepsilon)=\sum_{n}\left(\sum_{k_{1}, \ldots, k_{n} \in \mathbb{N}^{*}} \frac{\beta_{k_{1}} * \cdots * \beta_{k_{n}}}{k_{1}\left(k_{1}+k_{2}\right) \ldots\left(k_{1}+\cdots+k_{n}\right)}\right) \frac{1}{\varepsilon^{n}}
$$

implying $\psi_{\beta} \in G_{-}(A)$. Next we observe $\psi_{\beta}^{t}=\Gamma\left(\beta^{t} / \varepsilon\right)$, which follows simply from (14) and, on use of (11):

$$
\left(\psi_{\beta}^{t}\right)^{-1} * t \frac{\partial}{\partial t} \psi_{\beta}^{t}=\varepsilon\left(\psi_{\beta}^{t}\right)^{-1} * Y \psi_{\beta}^{t}=\varepsilon \psi_{\beta}^{t} \circ D=\varepsilon \Gamma\left(\beta^{t} / \varepsilon\right) \circ D=\beta^{t} .
$$

Now, the BWH decomposition $\psi_{\beta}^{t}=\left(\psi_{\beta}^{t}\right)_{-}^{-1} *\left(\psi_{\beta}^{t}\right)_{+}$is such that :

$$
\begin{aligned}
\left(\psi_{\beta}^{t}\right)^{-1} * t \frac{\partial}{\partial t} \psi_{\beta}^{t} & =\left(\psi_{\beta}^{t}\right)^{-1} * t \frac{\partial}{\partial t}\left(\psi_{\beta}^{t}\right)_{-}^{-1} *\left(\psi_{\beta}^{t}\right)_{+}+\left(\psi_{\beta}^{t}\right)^{-1} *\left(\psi_{\beta}^{t}\right)_{-}^{-1} * t \frac{\partial}{\partial t}\left(\psi_{\beta}^{t}\right)_{+} \\
& =\left(\psi_{\beta}^{t}\right)_{+}^{-1} *\left(\psi_{\beta}^{t}\right)_{-} * t \frac{\partial}{\partial t}\left(\psi_{\beta}^{t}\right)_{-}^{-1} *\left(\psi_{\beta}^{t}\right)_{+}+\left(\psi_{\beta}^{t}\right)_{+}^{-1} * t \frac{\partial}{\partial t}\left(\psi_{\beta}^{t}\right)_{+} ;
\end{aligned}
$$

hence we find:

$$
\left(\psi_{\beta}^{t}\right)_{+} * \beta^{t} *\left(\psi_{\beta}^{t}\right)_{+}^{-1}=\left(\psi_{\beta}^{t}\right)_{-} * t \frac{\partial}{\partial t}\left(\psi_{\beta}^{t}\right)_{-}^{-1}+t \frac{\partial}{\partial t}\left(\psi_{\beta}^{t}\right)_{+} *\left(\psi_{\beta}^{t}\right)_{+}^{-1} .
$$

Using $\psi_{\beta} \in G_{-}(A)$ and that $\beta^{t}$ takes values in $A_{+}$, we find by applying the projector $\mathcal{R}_{-}$on both sides of the last equation :

$$
\mathcal{R}_{-}\left(\left(\psi_{\beta}^{t}\right)_{+} * \beta^{t} *\left(\psi_{\beta}^{t}\right)_{+}^{-1}\right)=0=\left(\psi_{\beta}^{t}\right)_{-} * t \frac{\partial}{\partial t}\left(\psi_{\beta}^{t}\right)_{-}^{-1}=-t \frac{\partial}{\partial t}\left(\psi_{\beta}^{t}\right)_{-} *\left(\psi_{\beta}^{t}\right)_{-}^{-1}
$$

implying that $\left(\psi_{\beta}^{t}\right)_{-}$is independent of $t$; thus $\Gamma(\beta / \varepsilon)$ is a local character.

The last proposition is suggestive of the fact that local $A$-valued characters are essentially determined by ordinary (scalar-valued) infinitesimal characters (beta functions). This is indeed the case. Before proving the main result of this section, we remark that, for any $\phi \in G^{\mathrm{loc}}(A)$, we can write :

$$
\phi^{t}=\phi_{-}^{-1} *\left(\phi^{t}\right)_{+}=\phi * h_{\phi}^{t},
$$

where $h_{\phi}^{t}:=\left(\phi_{+}\right)^{-1} *\left(\phi^{t}\right)_{+} \in G_{+}(A)$. Also, for $\phi \in G_{-}^{\text {loc }}(A)$ we denote $\phi_{:-1}$ by Res $\phi$.

Theorem 7.2. The map $\phi \mapsto \varepsilon(\phi \circ D)$, with $D$ the Dynkin operator, sends $G^{\text {loc }}(A)$ to $\Xi\left(A_{+}\right)$ and $G_{-}^{\mathrm{loc}}(A)$ to $\Xi(\mathbb{C})$; explicitly, in the second case:

$$
G_{-}^{\text {loc }}(A) \ni \phi \mapsto \varepsilon(\phi \circ D)=Y \operatorname{Res} \phi \in \Xi(\mathbb{C}) .
$$


Proof. First, recall from Corollary 4.2 that $D$ sends characters to infinitesimal characters and that by Proposition 7.1 any local $\phi \in G^{\mathrm{loc}}(A)$ decomposes as $\phi^{a} * \phi^{b}$ with $\phi^{a} \in G_{-}^{\mathrm{loc}}(A), \phi^{b} \in$ $G_{+}(A)$. Therefore, we see that:

$$
\phi \circ D=\phi^{-1} * Y \phi=\left(\phi^{b}\right)^{-1} *\left(\phi^{a}\right)^{-1} * Y \phi^{a} * \phi^{b}+\left(\phi^{b}\right)^{-1} * Y \phi^{b} ;
$$

since $\left(\phi^{b}\right)^{-1}$ and $Y \phi^{b}$ belong to $\operatorname{Lin}\left(H, A_{+}\right)$, the theorem follows if we can prove that:

$$
\epsilon(\phi \circ D)=\epsilon\left(\phi^{-1} * Y \phi\right) \in \Xi(\mathbb{C})
$$

when $\phi \in G_{-}^{\text {loc }}(A)$. Assume the latter is the case and recall the decomposition $\phi^{t}=\phi * h_{\phi}^{t}$ in (15), with now simply $h_{\phi}^{t}=\left(\phi^{t}\right)_{+}$. So that on the one hand (11) implies :

$$
\left.t \frac{\partial}{\partial t}\right|_{t=1} h_{\phi}^{t}=\left.\phi^{-1} * t \frac{\partial}{\partial t}\right|_{t=1} \phi^{t}=\phi^{-1} * \varepsilon Y \phi=\varepsilon(\phi \circ D) .
$$

On the other hand, observe that by using the Bogoliubov formula (6) one finds:

$$
\begin{aligned}
\left.t \frac{\partial}{\partial t}\right|_{t=1} h_{\phi}^{t} & =\left.t \frac{\partial}{\partial t}\right|_{t=1}\left(\phi^{t}\right)_{+}=\left.t \frac{\partial}{\partial t}\right|_{t=1} \mathcal{R}_{+}\left(\phi_{-} *\left(\phi^{t}-e\right)\right) \\
& =\left.t \frac{\partial}{\partial t}\right|_{t=1} \mathcal{R}_{+}\left(\phi^{-1} * \phi^{t}-\phi^{-1}\right)=\left.t \frac{\partial}{\partial t}\right|_{t=1} \mathcal{R}_{+}\left(\phi^{-1} * t^{\varepsilon \mid \cdot} \phi\right) \\
& =\mathcal{R}_{+}(\varepsilon Y \phi)=Y \operatorname{Res} \phi .
\end{aligned}
$$

In the step before the last we took into account that $Y\left(1_{H}\right)=0$ and that $\phi_{-} \in G_{-}^{\text {loc }}(A)$ which implies for $h \in H^{+}$:

$$
\phi^{-1} * t^{\varepsilon|\cdot|} \phi(h)=\phi^{-1}(h)+t^{\varepsilon|h|} \phi(h)+\phi^{-1}\left(\bar{h}^{(1)}\right) t^{\varepsilon\left|\bar{h}^{(2)}\right|} \phi\left(\bar{h}^{(2)}\right),
$$

where $h^{(1)} \otimes h^{(2)}=h \otimes 1+1 \otimes h+\bar{h}^{(1)} \otimes \bar{h}^{(2)}$. Here $\phi^{-1}(h) \in A_{-}$and $\phi^{-1}\left(\bar{h}^{(1)}\right) t^{\varepsilon \mid \bar{h}^{(2)}} \mid \phi\left(\bar{h}^{(2)}\right)$ are both mapped to zero by $\left.t \frac{\partial}{\partial t}\right|_{t=1} \mathcal{R}_{+}$.

A glance back to Theorem 4.1 and Proposition 7.2 allows us to conclude that for $\phi \in G_{-}^{\text {loc }}(A)$ one has:

$$
\Gamma\left(\frac{Y \operatorname{Res} \phi}{\varepsilon}\right)=\phi
$$

so indeed the polar part of a local character $\phi$ can be retrieved from its beta function, $\beta(\phi):=$ $Y \operatorname{Res} \phi \in \Xi(\mathbb{C})$, by the universal formula (1).

Equation (18) is equivalent to the 'scattering type formula' of [19]; we can refer to [42] for this. Now it is an easy task to recover the other results of [19,21]. We ought to remark that, for $\gamma$ a general local character, one defines $\operatorname{Res} \gamma=-\operatorname{Res} \gamma_{-} \longrightarrow$ see in this respect formulae [19, Equation (11)] or [21, Equation (2.111)].

Theorem 7.3. For the renormalized character $\gamma_{\mathrm{ren}}(t):=\left(\gamma^{t}\right)_{+}(\varepsilon=0)$ it holds:

$$
t \frac{\partial}{\partial t} \gamma_{\mathrm{ren}}(t)=(Y \operatorname{Res} \gamma) * \gamma_{\mathrm{ren}}(t)
$$

the abstract $R G$ equation.

Proof. First, in the proof of Theorem 7.2 we saw already that $D$ verifies a cocycle condition [28]: for $\phi, \psi \in G(A)$ :

$$
(\phi * \psi) \circ D=\psi^{-1} *(\phi \circ D) * \psi+\psi \circ D .
$$


This together with Theorem 7.2 implies for $\phi \in G_{-}^{\text {loc }}(A)$ that $\operatorname{Res} \phi=-\operatorname{Res} \phi^{-1}$. Indeed, this follows by taking the residue Res on both sides of the equation:

$$
0=\left(\phi^{-1} * \phi\right) \circ D=\phi^{-1} *\left(\phi^{-1} \circ D\right) * \phi+\phi \circ D=\phi^{-1} * \frac{\operatorname{Res} \phi^{-1}}{\varepsilon} * \phi+\frac{\operatorname{Res} \phi}{\varepsilon} .
$$

Now, let $\gamma \in G^{\text {loc }}(A)$ with BWH decomposition $\gamma^{t}=\gamma_{-}^{-1} *\left(\gamma^{t}\right)_{+}$. Recall that $\left(\gamma^{t}\right)_{+}=$ $\mathcal{R}_{+}\left(\gamma_{-} * t^{\varepsilon|\cdot|} \gamma\right)$ maps $H^{+}$into $A_{+} \otimes \mathbb{C}[[\log (t)]]$ such that:

$$
t \frac{\partial\left(\gamma^{t}\right)_{+}}{\partial t}(0)=t \frac{\partial}{\partial t} \gamma_{\mathrm{ren}}
$$

As $\gamma_{-} \in G_{-}^{\text {loc }}(A)$, we then find:

$$
\begin{aligned}
t \frac{\partial\left(\gamma^{t}\right)_{+}}{\partial t} & =\gamma_{-} * t \frac{\partial}{\partial t} \gamma^{t}=\gamma_{-} * \varepsilon Y \gamma^{t}=\gamma_{-} * \varepsilon Y\left(\gamma_{-}^{-1} *\left(\gamma^{t}\right)_{+}\right) \\
& =\gamma_{-} * \varepsilon Y\left(\gamma_{-}^{-1}\right) *\left(\gamma^{t}\right)_{+}+\varepsilon Y\left(\gamma^{t}\right)_{+}=\varepsilon\left(\gamma_{-}^{-1} \circ D\right) *\left(\gamma^{t}\right)_{+}+\varepsilon Y\left(\gamma^{t}\right)_{+} \\
& =\left(Y \operatorname{Res} \gamma_{-}^{-1}\right) *\left(\gamma^{t}\right)_{+}+\varepsilon Y\left(\gamma^{t}\right)_{+}=-\left(Y \operatorname{Res} \gamma_{-}\right) *\left(\gamma^{t}\right)_{+}+\varepsilon Y\left(\gamma^{t}\right)_{+} \\
& =(Y \operatorname{Res} \gamma) *\left(\gamma^{t}\right)_{+}+\varepsilon Y\left(\gamma^{t}\right)_{+}
\end{aligned}
$$

Therefore both sides have a limit as $\varepsilon \downarrow 0$, yielding the sought after RG equation (19).

Equation (19) is solved using the beta function $\beta(\gamma):=Y \operatorname{Res} \gamma \in \Xi(\mathbb{C})$ :

$$
\gamma_{\mathrm{ren}}(t)=\exp (\ln (t) \beta(\gamma)) * \gamma_{\mathrm{ren}}(1)
$$

The last statement and equation (13) tell us that:

$$
\lim _{\varepsilon \rightarrow 0} \gamma_{-} *\left(\gamma_{-}^{-1}\right)^{t}=\lim _{\varepsilon \rightarrow 0}\left(\gamma^{t}\right)_{+} *\left(\left(\gamma_{+}\right)^{t}\right)^{-1}=\gamma_{\mathrm{ren}}(t) * \gamma_{\mathrm{ren}}^{-1}(1)=\exp (\ln (t) \beta(\gamma)) .
$$

The scalar-valued characters

$$
\Omega_{t}(\gamma):=\exp (\ln (t) \beta(\gamma)) \in G(\mathbb{C})
$$

obviously form a one-parameter subgroup in $G(A): \Omega_{t_{1}}(\gamma) * \Omega_{t_{2}}(\gamma)=\Omega_{t_{1} t_{2}}(\gamma)$, generated by the beta function and controlling the flow of the renormalized Feynman rule character with respect to the mass scale.

\section{Through the PRISM of Other RENORMALIZATION SCHEMES I}

We plan now to prospect the usefulness of our approach in other schemes of renormalization. Doubtless DR provides the cleanest formulation of locality in the BWH decomposition for renormalization. However, it is physically clear that in any scheme one has still to parameterize the arbitrariness in separating divergent from finite parts; and that the physical irrelevance of the choices made still gives rise to the RG equation. On the mathematical side, it is worth to recall that the algebraic BWH decomposition of Section 5 is not necessarily linked to loops on the Riemann sphere. It is thus legitimate to ponder the question in schemes other than those based on DR. We plan to exemplify with the BPHZ scheme in the next section, but, before dwelling on that, let us briefly indicate other pieces of evidence.

A first one concerns old research. In the early seventies, Callan set out to prove that broken scale invariance [10] is all that renormalization was about. He was eventually able to give a treatment of the RG, and proofs of renormalizability of field theories based on the former, by relying entirely in the BPHZ formalism. To derive the beta function, he just set up RG equations by studying the dependency of the $N$-point functions on the renormalized mass. See in this respect $[4,11]$. In a renormalization method without regularization, information about the RG must be stored somehow in the renormalized quantities. Concretely, as hinted 
at by our last theorem, one finds it in the scaling properties of the renormalized integral. This was noted in the context of Epstein-Glaser renormalization in [33]. In DR this shows in the RG equation (19).

A second piece of evidence is furnished by more recent work by Kreimer and collaborators [3, $8,38,40]$. Indeed, Kreimer has long argued that locality (and renormalizability) is determined by the Hochschild cohomology of renormalization Hopf algebras. This cohomology is trivial in degree greater than one. The coproduct on $H$ can be written recursively in terms of closed Hochschild 1-cochains. Those are grafting maps indexed by primitive 1PI diagrams, that govern the structure of Feynman graphs and give rise through the Feynman rules to integral Dyson-Schwinger equations. Here is not the place for details and we refer the reader to $[3,8,37,38,40]$, and especially Kreimer's recent review [39]. The interaction between the Hopf algebra of characteristic functions of $H$ of this paper and the Hochschild 1-cocycles on $H$ is a promising field of interest.

In the indicated references the Dynkin operator $D$ (and its close cousins $S * Y^{n}$ ) appears, again defining the residue, in renormalization schemes without regularization. There Green's functions, $\Sigma=\Sigma(g, p)$, are defined in terms of their (combinatorial) Dyson-Schwinger equations using the Hochschild 1-cocycles; $g, p$ denote the coupling constant and external momenta, respectively. Those Green's functions are expanded as power series in $g$ :

$$
\Sigma=1+\sum_{k>0} \phi\left(c_{k}\right) g^{k}
$$

for Feynman rules $\phi \in G(\mathbb{C})$ and with order by order divergent Feynman amplitudes $\phi\left(c_{k}\right)$ as coefficients. Here the $c_{k}$ 's are particular linear combinations of graphs of loop order $k$ in $H$ [37]. Renormalization of $\Sigma$ is achieved by using a renormalized character $\phi_{\text {ren }} \in G(\mathbb{C})$ defined by subtraction at a specific point $p^{2}=\lambda^{2}$-corresponding to Taylor expansion up to zeroth order. Here $\lambda$ plays the role of the mass-parameter $\mu$. Locality is automatically fulfilled in this approach. The renormalized Green's functions $\Sigma_{\text {ren }}=\Sigma_{\text {ren }}(g, p, \lambda)$ can be developed in terms of the parameter $L:=\log \left(p^{2} / \lambda^{2}\right)$, hence $\Sigma_{\text {ren }}=1+\sum_{k>0} \alpha_{k}(g) L^{k}$, with $\alpha_{1}(g) \in \Xi(\mathbb{C})[40]$. Following the above references and adapting partially to our notation, the residue is found to be:

$$
\Xi(\mathbb{C}) \ni \sigma_{1}:=\left.\frac{\partial}{\partial L}\left(\phi_{\text {ren }} \circ D\right)\right|_{L=0}=\alpha_{1}(g) .
$$

In [40] Kreimer and Yeats outline how to derive $\alpha_{k}(g), k>1$ recursively from $\alpha_{1}(g)$. This confirms that, in a deep sense, the beta function is composition with the Dynkin operator.

\section{Through the Prism of Other Renormalization SCHEMES II}

Let us now explore the classical BPHZ scheme in the context of the former sections. With $I_{F}$ the integrand of (17) corresponding to the graph $F$, let us write for the Taylor subtraction employed in BPHZ renormalization:

$$
I_{F}(p, k) \mapsto I_{F}(p, k)-t_{p}^{s(F)} I_{F}(p, k):=I_{F}(p, k)-\sum_{|\alpha| \leq s(F)} \frac{p^{\alpha}}{\alpha !} \partial_{\alpha} I_{F}(0, k) .
$$

We borrowed here the standard multi-index notation

$$
\alpha=\left\{\alpha_{1}, \ldots, \alpha_{n}\right\} \in \mathbb{N}^{n}, \quad|\alpha|:=\sum_{i=1}^{n} \alpha_{i}, \quad \alpha !=\prod_{i=1}^{n} \alpha_{i} !
$$


each $\alpha_{i}$ takes values between 0 and 3 , say. We are assuming that only massive particles are present, otherwise the subtraction at zero momentum gives rise to infrared divergences; the expression of $t_{p}^{s(F)} I_{F}$ in practice simplifies because of Lorentz invariance.

Notice that the integral $J_{F}(p)$ in (7) does originally have a meaning: it is a well defined functional on the linear subspace $\mathcal{S}_{s(F)}\left(\mathbb{R}^{4 N}\right)$ of Schwartz functions $\phi$ on the external momenta, whose first moments $\int p^{\alpha} \phi(p) d^{4 N} p$ up to order $|\alpha| \leq s(F)$ happen to vanish. The "divergent" loop integrals inside $J_{F}(p)$ become harmless when coupled exclusively with Schwartz functions of this type. The Taylor 'jet' projector map $t_{p}^{l}$ subtracts polynomials, that are functionals of the same type, in such a way that the result (eventually) becomes moreover a tempered distribution.

The first question is whether we have a Rota-Baxter algebra in the BPHZ context. Actually, we do have the Rota-Baxter property for $t_{p}^{l}$. Indeed, the following is obtained by a simple calculation from the definitions.

Proposition 9.1. Let $I_{F_{i}}, i=1,2$ have associated degrees of divergence $l_{i}, i=1,2$. Then

$$
t_{p_{1}}^{l_{1}}\left(I_{F_{1}}\right) t_{p_{2}}^{l_{2}}\left(I_{F_{2}}\right)=t_{p_{1}, p_{2}}^{l_{1}+l_{2}}\left(I_{F_{1}} t_{p_{2}}^{l_{2}}\left(I_{F_{2}}\right)\right)+t_{p_{1}, p_{2}}^{l_{1}+l_{2}}\left(t_{p_{1}}^{l_{1}}\left(I_{F_{1}}\right) I_{F_{2}}\right)-t_{p_{1}, p_{2}}^{l_{1}+l_{2}}\left(I_{F_{1}} I_{F_{2}}\right) .
$$

We leave the verification of this to the care of the reader. In general, if $U$ is a multiplicative semigroup, a family of linear operators $R_{u}, u \in U$ on the algebra $A$ is called a Rota-Baxter family if for any $u, v \in U$ and $a, b \in A$, we have

$$
R_{u}(a) R_{v}(b)=R_{u v}\left(a R_{v}(b)\right)+R_{u v}\left(R_{u}(a) b\right)-R_{u v}(a b), \quad \text { for all } \quad a, b \in A .
$$

Thus the $l$-jets define a Rota-Baxter family. Now, a Rota-Baxter family is almost the same thing as a Rota-Baxter operator.

Proposition 9.2. Let $\mathcal{A}=A[U]$ be the semigroup algebra associated to $A$. Let $R_{u}: A \rightarrow$ $A, u \in U$ be a Rota-Baxter family. Define

$$
R: \mathcal{A} \rightarrow \mathcal{A}, \quad \text { by } \quad R\left(\sum_{u} a_{u} u\right):=\sum_{u} R_{u}\left(a_{u}\right) u .
$$

Then $R$ is a Rota-Baxter operator on $\mathcal{A}$ such that $R(a u)=a^{\prime} u$ with $a^{\prime}$ in $A$. Conversely, if $R: \mathcal{A} \rightarrow \mathcal{A}$ is a Rota-Baxter operator such that $R(a u)=a^{\prime} u$ with $a^{\prime}$ in $A$, then we obtain a Rota-Baxter family $R_{u}, u \in U$, by defining $R_{u}(a)=a^{\prime}$ where $R(a u)=a^{\prime} u$.

The proof is immediate 1 . On the strength of the previous result, we may refer to a RotaBaxter family as a Rota-Baxter operator. Now, pQFT in practice furnishes an even more radical answer to the question of whether one has here the Rota-Baxter framework. For this is obviously the case when one deals only with logarithmic divergences; and indeed most often only the latter is required. In general, differentiation of an amplitude with respect to an external momentum lowers the overall degree of divergence of a diagram. In DR, the CaswellKennedy theorem [13] states that the pole part of any diagram, besides being independent of the scale, is a polynomial in the external momentum. This follows easily from that derivation and the projector $R_{-}$commute in DR. But even in the BPHZ scheme $\partial_{p} t^{l}=t^{l-1} \partial_{p}$, and this is enough for the differentiation trick to work.

Let us then consider the $J_{F}(p) \in \mathcal{S}_{s(F)}^{\prime}\left(\mathbb{R}^{4 N}\right)$ of (17). Suppose moreover the multi-loop divergent graph $F$ has all its 1PI subgraphs $\gamma$ made convergent by application of Bogoliubov's

\footnotetext{
${ }^{1}$ We thank L. Guo for suggesting the notion of Rota-Baxter family
} 
preparation map $\bar{R}$. Then the renormalized integral $J_{F}^{\mathrm{ren}, \mathrm{BPHZ}}(p)$ can be defined as

$$
J_{F}^{\mathrm{ren}, \mathrm{BPHZ}}(p)=\left[\int \prod_{l=1}^{|F|} d^{4} k_{l}\right]\left(I_{F}(p, k)-t_{p}^{s(F)} \bar{R} I_{F}(p, k)\right)=:\left[\int \prod_{l=1}^{|F|} d^{4} k_{l}\right] R_{F}(p, k) .
$$

This recipe is however not unique. We can write as well

$$
J_{F}^{\mathrm{ren}, \mathrm{BPHZ}}(p)=P^{s(F)}(p)+\left[\int \prod_{l=1}^{|F|} d^{4} k_{l}\right] R_{F}(p, k),
$$

with $P^{s(F)}$ a polynomial of order $s(F)$ in the external momenta. This effects a 'finite renormalization', in principle undetermined, that might be put to use to fulfil renormalization prescriptions (again, the form of that polynomial is severely restricted by Lorentz invariance).

We now come to the key point. The coefficients of $P^{s(F)}$ in (21) exhibit the ambiguity of renormalization in the context of the BPHZ scheme. On the face of it, the 'pole terms' $t_{p}^{s(F)} I_{F}(p, k)$ do not depend at all on the mentioned coefficients, and thus locality of the BWH decomposition is guaranteed, in a trivial sense. On the other hand, the Galois group approach to renormalization theory $[20,21]$ stems originally from the idea that ambiguities should be, insofar as possible, handled from a group-theoretic point of view, much as classical Galois theory handles the multiple solutions of polynomial equations. Here however the mentioned form of the ambiguity does not apparently lend itself to RG analysis. We contend, however, that the ambiguity is expressed essentially in the same form as before. The Caswell-Kennedy theorem is suggestive of a direct link between the DR and BPHZ formalisms, and next we endeavour to prove the pertinence of the $\mathrm{RG}$ to $\mathrm{BPHZ}$ renormalization by the most direct possible route: introducing a mass scale in the latter formalism in direct analogy with the former.

To express the ambiguity implicit in the $P^{s(F)}$ of (21) in terms of a mass scale, we use the modified BPHZ scheme proposed in [29]. For instance, it is well known that the famous graph $\bigcirc$ ('fish' graph) giving the first nontrivial contribution to the vertex correction in the $\varphi_{4}^{4}$ model in the Euclidean yields the amplitude

$$
J_{\mathrm{fish}}^{\mathrm{DR}}(p)=\tilde{g}^{2} \mu^{2 \varepsilon} \int \frac{d^{\mathrm{D}} k}{(2 \pi)^{4}} \frac{1}{k^{2}+m^{2}} \frac{1}{(p+k)^{2}+m^{2}},
$$

where $p=p_{1}+p_{2}$, say, and that, by use of Feynman's parametrization (see below) and relation (9) one obtains

$$
J_{\text {fish }}^{\mathrm{DR}}(p)=g \frac{\tilde{g}}{(4 \pi)^{2}}\left[\frac{2}{\varepsilon}+\int_{0}^{1} d z \log \frac{\bar{\mu}^{2}}{p^{2} z(1-z)+m^{2}}+O(\varepsilon)\right] .
$$

Now, the natural 'zero point' for the mass scale in this problem is clearly $m$, and we note

$$
R_{+}\left(J_{\text {fish }}^{\mathrm{DR}}(p=0 ; \bar{\mu}=m)\right)=0,
$$

as $\varepsilon \downarrow 0$. This, together with the mentioned Caswell-Kennedy theorem, feeds the suspicion that the last expression is just the $J_{\text {fish }}^{\text {ren } B P H Z}(p)$ of (20). The suspicion is correct. The computation required for the renormalized fish graph in the BPHZ scheme is

$$
g^{2} \int \frac{d^{4} k}{(2 \pi)^{4}}\left(1-t_{p}^{0}\right)\left(\frac{1}{k^{2}+m^{2}} \frac{1}{(p+k)^{2}+m^{2}}\right) .
$$


Introduce the Feynman trick, prior to the Taylor subtraction,

$$
\begin{aligned}
& g^{2} \int_{0}^{1} d z \int \frac{d^{4} k}{(2 \pi)^{4}}\left(1-t_{p}^{0}\right) \frac{1}{\left[\left((p+k)^{2}+m^{2}\right) z+(1-z)\left(k^{2}+m^{2}\right)\right]^{2}} \\
& =g^{2} \int_{0}^{1} d z \int \frac{d^{4} k}{(2 \pi)^{4}}\left(1-t_{p}^{0}\right) \frac{1}{\left[k^{2}+p^{2} z(1-z)+m^{2}\right]^{2}} .
\end{aligned}
$$

The translation $k \rightarrow k-z p$, depending on the Feynman parameter, has been made in order to obtain here the same denominator as in DR calculations. With $\Omega_{4}$ the area of the unit sphere in $\mathbb{R}^{4}$, the integral (23) now becomes

$$
\begin{aligned}
& \frac{\Omega_{4} g^{2}}{(2 \pi)^{4}} \int_{0}^{1} d z \int_{0}^{\infty} d k\left[\frac{k^{3}}{\left[k^{2}+p^{2} z(1-z)+m^{2}\right]^{2}}-\frac{k^{3}}{\left[k^{2}+m^{2}\right]^{2}}\right] \\
& =\frac{g^{2}}{16 \pi^{2}} \int_{0}^{1} d z \log \frac{m^{2}}{p^{2} z(1-z)+m^{2}} .
\end{aligned}
$$

The last step is to convert the $p$-independent part in the argument of the logarithm into a mass scale: $m \rightarrow \bar{\mu}$. With this, we recover on the nose the DR result, in the $\overline{\mathrm{MS}}$ scheme as it turns out. Incidentally, as remarked in [46], this procedure allows us to give the exact value of the BPHZ integral (22): the expression $\int_{0}^{1} d z \log \left(1+\frac{p^{2}}{m^{2}} z(1-z)\right)$ is actually well known in statistical physics, and leads by elementary manipulations involving the golden ratio to

$$
J_{\text {fish }}^{\text {ren,BPHZ }}(p)=-\frac{g^{2}}{16 \pi^{2}}\left(\sqrt{1+\frac{4 m^{2}}{p^{2}}} \log \frac{\sqrt{4 m^{2} / p^{2}}+1}{\sqrt{4 m^{2} / p^{2}}-1}-2\right) .
$$

Thus, what we have done above amounts to identify the constant term - recall $P^{s(F)}(p)$ in (21). We have the right to add to the previous expression the term $g^{2} / 16 \pi^{2}$ times $\log \left(\bar{\mu}^{2} / m^{2}\right)$. We note also that one can recover the residue $g^{2} / 8 \pi^{2}$ here from $J_{\text {fish }}^{\text {ren,BPHZ }}$, as the coefficient of the term logarithmic in the scaling factor,

$$
J_{\text {fish }}^{\mathrm{ren}, \mathrm{BPHZ}}(\lambda p) \sim J_{\text {fish }}^{\mathrm{ren}, \mathrm{BPHZ}}(p)-\frac{g^{2}}{8 \pi^{2}} \log \lambda,
$$

as $\lambda \uparrow \infty$.

The steps of the modified BPHZ procedure are: (i) Introduction of the Feynman parametrization in $J_{F}^{\mathrm{BPHZ}}(k, p)$. (ii) Exchange of the integrations. (iii) Translation of the integration variables by $\lambda p$, with $\lambda$ dependent on the Feynman parameter. (iv) Taylor subtraction. (v) Integration over loops and replacement of the mass $m$ in the $p$-constant part of the resulting logarithm by a mass scale. There is nothing to forbid the same operations to be performed on any primitive logarithmically divergent graph of any field theory and then we are optimistic that, by use of skeletal expansions and the integral equations, we would be led to a procedure largely parallel to DR, and so to a brute-force proof that the coefficients of the higher powers of the scaling logarithms in BPHZ renormalization are determined by the residues. To verify this with full particulars, however, would take us too far afield.

Recapitulating, the Lie-theoretic method shows promise in dealing with renormalization schemes others than DR with MS. The presence of a Rota-Baxter structure is a requisite for the validity of such a framework; it obviously holds for the $\overline{\mathrm{MS}}$ prescription in DR as well. What of other renormalization methods? For massive fields, the BPHZ scheme does verify the required conditions. We have learned, however, that the details are very idiosyncratic: as stated above, locality is moot; and the beta function enters the picture through Theorem 7.3 , referring to renormalized quantities, rather than to counterterms. For massless fields, the price 
of the Rota-Baxter property is relinquishing Lorentz invariance, and this is too heavy to pay. The Taylor subtraction in Epstein-Glaser renormalization has roughly the same properties as the BPHZ scheme, both in regard of massive and massless fields; nevertheless, there one is confronted to the problems of good definition that plague the attempts $[2,32]$. Procedures based on analytic renormalization or Hadamard regularization [33] have not been investigated yet from the Rota-Baxter viewpoint. Thus it is too early in the game to draw a list of known schemes that would definitely fit in our approach; we plan to come to this in future work. It is intriguing that the case study of $\mathrm{BPHZ}$ renormalization points out to the pertinence of the $\overline{\mathrm{MS}}$ prescription in DR.

\section{On Connes-Marcolli's motivic Galois theory}

In the Connes-Kreimer picture of renormalization, group theory and the scheme-theoretic equivalent theory of commutative Hopf algebras have become a fundamental tool of pQFT. Connes and Marcolli identified recently [20] a new level at which Hopf algebra structures enter pQFT. Namely, they constructed an affine group scheme $U^{*}$, universal with respect to physical theories, and pointed out its analogies with number theory, e.g. with the motivic Galois group of the scheme of 4 -cyclotomic integers $\mathbb{Z}[i]\left[\frac{1}{2}\right]$.

In their work the initial physical problem attacked through the Connes-Kreimer paradigm translates into the classification of equisingular $G$-valued flat connections on the total space of a principal bundle over an infinitesimal punctured disk (with $G$ the group scheme represented by $H$ ). From the representation theoretic point of view, the classification is provided by representations $U^{*} \longrightarrow G^{*}$, where $U^{*}$ is the semi-direct product with the grading of the prounipotent group $U$, the Lie algebra of which is the free graded Lie algebra with one generator $e_{n}$ in each degree $n>0$, and similarly for $G^{*}$. Returning to the geometrical side of the correspondence and featuring the DR setting that leads to the Riemann-Hilbert picture of renormalization, Connes and Marcolli construct a universal singular frame on principal $U$ bundles over $B$. A formal expression for it is given by:

$$
\gamma(\varepsilon, v)=\sum_{n \geq 0} \sum_{k_{j}} \frac{e\left(k_{1}\right) \cdots e\left(k_{n}\right)}{k_{1}\left(k_{1}+k_{2}\right) \cdots\left(k_{1}+\cdots+k_{n}\right)} v^{\sum k_{j}} \varepsilon^{-n} .
$$

As already remarked in [20] and our introduction, it is interesting that the coefficients of the frame are essentially those appearing in the index formula of Connes-Moscovici; this would hint at the rooting of noncommutative geometry in quantum field theory, which has been Connes' contention for a long while.

We have already shown that other Hopf algebra structures (or, from the scheme-theoretic point of view, pro-unipotent groups) do appear naturally in pQFT, namely the Hopf algebras $\operatorname{Char}(A)$ of characteristic functions associated to commutative target algebras, e.g., although not exclusively, of quantum amplitudes. These Hopf algebra structures arise naturally from algebraic-combinatorial constructions on Hopf algebras, and therefore do not immediately relate to the geometrical-arithmetical constructions underlying the definition of the motivic Galois group in [20]. Nevertheless, the formula rendering the universal singular frame in the motivic understanding of renormalization also essentially coincides with our map $\Gamma$ - the inverse of the Dynkin map. This indicates that the practical consequences for renormalization of the Riemann-Hilbert and/or motivic point of view can be translated to the setting of FLA theory — which, besides being more familiar to many, is independent of the geometry embedded in the DR scheme. As it turns out, the pro-unipotent groups/Hopf algebras Char $(H)$ and $\operatorname{Char}(A)$ are related naturally to the group $U$. In the remainder of the present section, we 
would like to make explicit how both viewpoints connect —although the reasons behind this connection certainly ought to be deepened.

Let us recall a few general facts from the theory of Solomon algebras — see [44,47] for details. Let $\sigma$ be a permutation in the symmetric group $S_{n}$ of order $n$. The descent set $D(\sigma)$ of $\sigma$ is the set $D(\sigma):=\{i, \sigma(i)>\sigma(i+1)\}$. Note $n \notin D(\sigma)$. The descent composition $C(\sigma)$ of $\sigma$ is the composition of $n$ (that is, the sequence $\left(c_{1}, \ldots, c_{k}\right)$ of strictly positive integers of total sum $n$ ) such that, when viewed as a word, $\sigma=\sigma(1) \ldots \sigma(n)$ can be written $u_{1} \ldots u_{k}$, where each word $u_{i}$ is increasing and of length $c_{i}$, and where $k$ is minimal. For example, $D(21534)=\{1,3\}$ and $C(21534)=(1,2,2)$. The notions of descent set and descent composition are obviously equivalent, the equivalence being induced by the map:

$$
\left(c_{1}, \ldots, c_{k}\right) \longmapsto\left\{c_{1}, c_{1}+c_{2}, \ldots, c_{1}+\cdots+c_{k-1}\right\} .
$$

The Solomon algebra $\Sigma_{n}$ of type $A_{n}$ was first introduced as a noncommutative lift to the group algebra of the representation ring of $S_{n}$ [50]. As a vector space, $\Sigma_{n}$ is the linear span of the elements $D_{\subseteq S}$ in $\mathbb{Q}\left[S_{n}\right]$, where $S$ runs over subsets of $[n-1]$ and

$$
D_{\subseteq S}:=\sum_{\substack{\sigma \in S_{n} \\ D(\sigma) \subseteq S}} \sigma .
$$

Then Solomon's fundamental theorem states that $\Sigma_{n}$ is closed under the composition product in $S_{n}$.

Now, let $X$ be an infinite alphabet. The dual graded Hopf algebra $T^{*}(X)=\bigoplus_{n \in \mathbb{N}} T_{n}^{*}(X)$ of $T(X)$ is graded connected commutative, with the shuffle product as the algebra product and the deconcatenation coproduct:

$$
x_{i_{1}} \ldots x_{i_{n}} \longmapsto \sum_{k=0}^{n} x_{i_{1}} \ldots x_{i_{k}} \otimes x_{i_{k+1}} \ldots x_{i_{n}},
$$

where we view $x_{i_{1}} \ldots x_{i_{n}}$ as an element of $T^{*}(X)$ using the usual pairing $\left\langle x_{i_{1}} \ldots x_{i_{n}} \mid x_{j_{1}} \ldots x_{j_{k}}\right\rangle=$ $\delta_{x_{i_{1}} \ldots x_{i_{n}}}^{x_{j_{1}} \ldots x_{j_{k}}}$. The symmetric group of order $n$ embeds into $\operatorname{End}\left(T_{n}^{*}(X)\right) \subset \operatorname{End}\left(T^{*}(X)\right)$ :

$$
\sigma\left(x_{i_{1}} \ldots x_{i_{n}}\right):=x_{i_{\sigma^{-1}(1)}} \ldots x_{i_{\sigma^{-1}(n)}} .
$$

This map induces an embedding of algebras of $\Sigma_{n}$ into $\operatorname{End}\left(T^{*}(X)\right)$, where the product on the latter algebra is the composition of maps.

Let us write now $\mathcal{D}$ for the descent algebra of $T^{*}(X)$, that is the convolution subalgebra of $\operatorname{End}\left(T^{*}(X)\right)$ generated by the projections $p_{n}: T^{*}(X) \longrightarrow T_{n}^{*}(X)$ on the graded components of $T^{*}(X)$. The algebra $\mathcal{D}$ is naturally graded and provided with a Hopf algebra structure for which the $p_{n}$ form a sequence of divided powers:

$$
\Delta\left(p_{n}\right)=\sum_{i+j=n} p_{i} \otimes p_{j}
$$

We write $\mathcal{D}_{n}$ for the component of degree $n$.

Lemma 10.1. The convolution algebra $\mathcal{D}$ is also closed under the composition of maps $\circ$ in $\operatorname{End}\left(T^{*}(X)\right)$.

The result follows from Corollary 9.4 in [47] (where the dual setting is considered, that is, the convolution subalgebra $\Gamma$ of $\operatorname{End}(T(X))$ generated by the graded projections in $T(X)$ ) and also follows directly from [44, Thm II.7]. 
Proposition 10.1. The embedding of $\Sigma_{n}$ into $\left(\operatorname{End}\left(T^{*}(X)\right), \circ\right)$ induces an isomorphism of algebras

$$
\Sigma_{n} \longrightarrow \mathcal{D}_{n}
$$

The proof follows from Corollary 9.2 in [47] by duality. It basically amounts to observe that, if $C(\sigma)=\left(c_{1}, \ldots, c_{k}\right)$, then $\sigma^{-1}$ is a $\left(c_{1}, \ldots, c_{k}\right)$-shuffle. For example, if $\sigma=(21534)$ then $C(\sigma)=(1,2,2)$ and $\sigma^{-1}=(21453)$, so that the word $\sigma\left(x_{1} \ldots x_{5}\right)=x_{2} x_{1} x_{4} x_{5} x_{3}$ is a shuffle of $x_{1}, x_{2} x_{3}$ and $x_{4} x_{5}$, and appears therefore in the expansion of $p_{1} * p_{2} * p_{2}\left(x_{1} \ldots x_{5}\right)$.

Proposition 10.2. The algebra $\mathcal{D}$ is freely generated as an associative algebra by the graded projections $p_{n}$. Equivalently, it is freely generated by the graded components $p_{n} \circ D$ of the Dynkin idempotent $D=S * Y$, regarded as an element of $\operatorname{End}\left(T^{*}(X)\right)$.

The first part of the proof of this proposition is Corollary 9.14 of [47] (stated for $\Gamma$, that is, in the dual setting). The second assertion is found e.g. in [31, Sect. 5.2].

Corollary 10.1. Regarded as a pro-unipotent group scheme, the graded dual Hopf algebra $\mathcal{D}^{*}$ is canonically isomorphic to the ring of coordinates of the Connes-Marcolli group $U$ of renormalization theory.

Through this correspondence, by our Lemma 4.1, the coefficients of the universal singular frame are reflected in the coefficients of the expansion of the identity of $T^{*}(X)$ on the natural linear basis of $\mathcal{D}$ viewed as the free associative algebra generated by the graded components of the Dynkin operator. Now, the universal properties of the Galois group $U$ for renormalization, when the group is understood by means of $\mathcal{D}$, follow from the constructions in [44], where it is shown that the descent algebra is an algebra of natural (endo)transformations of the forgetful functor from graded connected commutative Hopf algebras to graded vector spaces - that is, an universal endomorphism algebra for graded connected commutative Hopf algebras. In other terms, there is a natural map from $\mathcal{D}$ to $\operatorname{End}(H)$, where $H$ is an arbitrary graded connected commutative Hopf algebra. Using the arguments developed in the first sections of this article, one shows easily that this map factorizes through an Hopf algebra map from $\mathcal{D}$ to $\operatorname{Char}(H)$; this follows e.g. from the fact that the graded projections generate $\mathcal{D}$ as a convolution algebra and form a sequence of divided powers both in $\mathcal{D} \subset \operatorname{End}\left(T^{*}(X)\right)$ and in $\operatorname{Char}(H)$. In summary,

Corollary 10.2. The descent algebra $\mathcal{D}$ acts naturally by right composition on $\operatorname{Lin}(H, A)$. Moreover, the group of group-like elements in $\mathcal{D}$ acts naturally on the group $G(A)$ of Feynman rules.

The second part of the corollary follows from the third identity in Lemma 3.1 .

Besides providing a natural link between the Galoisian approach to renormalization and the noncommutative representation theory of the symmetric groups [5], the combinatorial approach implies moreover that the Connes-Marcolli universal Galois group $U$ inherits the very rich structure of the descent algebra. The appearance of the descent algebra (or equivalently of the Hopf algebras of noncommutative symmetric functions and quasi-symmetric functions, see [31]), beyond broadening the scope of the mathematical theory of renormalization, should result into new developments in the field, possibly complementary with the arithmetic ones.

\section{Acknowledgements}

The first named author 8 acknowledges greatly the support by the European Post-Doctoral Institute and the Institut des Hautes Études Scientifiques (I.H.É.S.). He is also indebted to

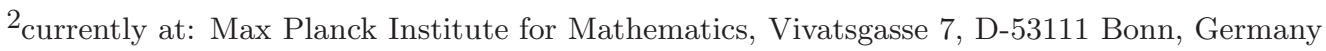


Laboratoire J. A. Dieudonné at Université de Nice Sophia-Antipolis for warm hospitality. He is very grateful to $\mathrm{C}$. Bergbauer for useful discussions. The second named author acknowledges partial support from CICyT, Spain, through grant FIS2005-02309. He is also grateful for the hospitality of Laboratoire J. A. Dieudonné. The present work received support from the ANR grant AHBE 05-42234. We are pleased to thank the anonymous referee, whose comments prompted us to clarify several aspects of the paper.

\section{REFERENCES}

[1] F. V. Atkinson, Some aspects of Baxter's functional equation, J. Math. Anal. Appl. 7 (1963) 1-30.

[2] C. Bergbauer and D. Kreimer, The Hopf algebra of rooted trees in Epstein-Glaser renormalization, Ann. Henri Poincaré 6 (2005) 343-367. ArXiv:hep-th/0403207.

[3] C. Bergbauer and D. Kreimer, Hopf Algebras in renormalization theory: locality and Dyson-Schwinger equations from Hochschild cohomology, in IRMA lectures in Mathematics and Theoretical Physics Vol. 10 (2006) 133-164, European Mathematical Society, Eds. V. Turaev, L. Nyssen. ArXiv:hep-th/0506190.

[4] A. S. Blaer and K. Young, Field theory renormalization using the Callan-Symanzik equation, Nucl. Phys. B 83 (1974) 493-514.

[5] D. Blessenohl and M. Schocker, Noncommutative character theory of the symmetric group, World Scientific, Singapore, 2005.

[6] S. Bloch, H. Esnault and D. Kreimer, Motives associated to graph polynomials, Commun. Math. Phys. 267 (2006) 181-225. ArXiv:math.ag/0510011

[7] D. J. Broadhurst and D. Kreimer, Towards cohomology of renormalization: bigrading the combinatorial Hopf algebra of rooted trees, Commun. Math. Phys. 215 (2000) 217-236. ArXiv:hep-th/0001202.

[8] D. J. Broadhurst and D. Kreimer, Exact solutions of Dyson-Schwinger equations for iterated one-loop integrals and propagator-coupling duality, Nucl. Phys. B 600 (2001) 403-422. ArXiv:hep-th/0012146.

[9] N. Bourbaki, Elements of Mathematics. Lie groups and Lie algebras. Chapters 1-3, Springer, Berlin, 1989.

[10] C. G. Callan, Broken scale invariance in scalar field theory, Phys. Rev. D 2 (1970) 1541-1547.

[11] C. G. Callan, Introduction to renormalization theory, in Methods in Field Theory, Les Houches 1975, North-Holland, Amsterdam, 1976, Eds. R. Balian, J. Zinn-Justin.

[12] P. Cartier, A primer on Hopf algebras, IHES preprint, August 2006.

[13] W. E. Caswell and A. D. Kennedy, Simple approach to renormalization theory, Phys. Rev. D 25 (1982) 392-408.

[14] J. C. Collins, Structure of the counterterms in dimensional regularization, Nucl. Phys. B 80 (1974) 341-348.

[15] J. C. Collins, Renormalization, Cambridge University Press, Cambridge, 1984.

[16] A. Connes and H. Moscovici, The local index formula in noncommutative geometry, Geom. Func. Anal. 5 (1995) 174-243.

[17] A. Connes and D. Kreimer, Hopf algebras, renormalization and noncommutative geometry, Commun. Math. Phys. 199 (1998) 203-242. ArXiv:hep-th/9808042.

[18] A. Connes and D. Kreimer, Renormalization in quantum field theory and the Riemann-Hilbert problem I. The Hopf algebra structure of graphs and the main theorem, Commun. Math. Phys. 210 (2000) 249-273. ArXiv:hep-th/9912092.

[19] A. Connes and D. Kreimer, Renormalization in quantum field theory and the Riemann-Hilbert problem. II. The $\beta$-function, diffeomorphisms and the renormalization group, Commun. Math. Phys. 216 (2001) 215-241. ArXiv:hep-th/0003188.

[20] A. Connes and M. Marcolli, Renormalization and motivic Galois theory. Internat. Math. Res. Notices Vol. 200476 (2004), 4073-4091. ArXiv:math.NT/0409306.

[21] A. Connes and M. Marcolli, From Physics to Number Theory via Noncommutative Geometry II: Renormalization, the Riemann-Hilbert correspondence, and motivic Galois theory, to appear in Frontiers in Number Theory, Physics and Geometry, ArXiv:hep-th/0411114.

[22] A. Connes and M. Marcolli, Quantum Fields and Motives, J. Geom. Phys. 56 (2006) 55-85. ArXiv:hep-th/0504085.

[23] K. Ebrahimi-Fard, L. Guo and D. Kreimer, Spitzer's identity and the algebraic Birkhoff decomposition in pQFT, J. Phys. A 37 (2004) 11037-11052. ArXiv:hep-th/0407082.

[24] K. Ebrahimi-Fard, L. Guo and D. Kreimer, Integrable Renormalization II: the General case, Ann. H. Poincaré 6 (2005) 369-395. ArXiv:hep-th/0402095. 
[25] K. Ebrahimi-Fard and D. Kreimer, Hopf algebra approach to Feynman diagram calculations, J. Phys. A 38 (2005) R385-R406. ArXiv:hep-th/0510202.

[26] K. Ebrahimi-Fard, J. M. Gracia-Bondía, L. Guo and J. C. Várilly, Combinatorics of renormalization as matrix calculus, Phys. Lett. B 632 (2006) 552-558. ArXiv:hep-th/0508154.

[27] K. Ebrahimi-Fard, L. Guo and D. Manchon, Birkhoff type decompositions and the Baker-CampbellHausdorff recursion, Commun. Math. Phys. 267 (2006) 821-845. ArXiv:math-ph/0602004.

[28] K. Ebrahimi-Fard and D. Manchon, On matrix differential equations in the Hopf algebra of renormalization, Adv. Theor. Math. Phys. 10 (2006) 879-913. ArXiv:math-ph/0606039.

[29] S. Falk, Doktor der Naturwissenschaften Dissertation, Mainz, 2005.

[30] H. Figueroa and J. M. Gracia-Bondía, Combinatorial Hopf algebras in quantum field theory I, Reviews of Mathematical Physics 17 (2005) 881-976. ArXiv:hep-th/0408145.

[31] I. M. Gelfand, D. Krob, A. Lascoux, B. Leclerc, V. Retakh and J.-Y. Thibon, Noncommutative symmetric functions, Adv. Math. 112 (1995) 218-348. ArXiv:hep-th/9407124.

[32] J. M. Gracia-Bondía and S. Lazzarini, Connes-Kreimer-Epstein-Glaser renormalization, ArXiv:hep-th/0006106.

[33] J. M. Gracia-Bondía, Improved Epstein-Glaser renormalization in coordinate space I. Euclidean framework, Math. Phys. Anal. Geom. 6 (2003) 59-88. ArXiv:hep-th/0202023.

[34] M. Hazewinkel, Hopf algebras of endomorphisms of Hopf algebras, ArXiv:math.QA/0410364.

[35] H. Kleinert and V. Schulte-Frohlinde, Critical Properties of $\phi^{4}$-theories, World Scientific, Singapore, 2001.

[36] D. Kreimer, On the Hopf algebra structure of perturbative quantum field theories, Adv. Theor. Math. Phys. 2 (1998) 303-334. ArXiv:q-alg/9707029.

[37] D. Kreimer, Anatomy of a gauge theory, Annals Phys. 321 (2006) 2757-2781. ArXiv:hep-th/0509135.

[38] D. Kreimer, Étude for linear Dyson-Schwinger Equations, IHES preprint, March 2006.

[39] D. Kreimer, Dyson-Schwinger Equations: from Hopf algebras to number theory, Fields Institute communications 50 (2007) 225-248. ArXiv:hep-th/0609004.

[40] D. Kreimer and K. Yeats, An Étude in non-linear Dyson-Schwinger Equations, ArXiv:hep-th/0605096.

[41] J. H. Lowenstein, BPHZ renormalization, in Renormalization theory (Proceedings NATO Advanced Study Institute, Erice, 1975), NATO Advanced Study Institute Series C: Math. and Phys. Sci. Vol. 23, Reidel, Dordrecht, 1976.

[42] D. Manchon, Hopf algebras, from basics to applications to renormalization, Comptes-rendus des Rencontres mathématiques de Glanon 2001, ArXiv:math.QA/0408405.

[43] F. Patras, La décomposition en poids des algèbres de Hopf, Ann. Inst. Fourier 43 (1993) 1067-1087.

[44] F. Patras, L'algèbre des descentes d'une bigèbre graduée, J. Algebra 170 (1994) 547-566.

[45] F. Patras and C. Reutenauer, On Dynkin and Klyachko idempotents in graded bialgebras, Adv. Appl. Math. 28 (2002) 560-579.

[46] O. Piguet and S. P. Sorella, Algebraic renormalization, Springer, Berlin, 1995.

[47] C. Reutenauer, Free Lie algebras, Oxford University Press, Oxford, 1993.

[48] S. Shnider and S. Sternberg, Quantum groups. From coalgebras to Drinfel'd algebras. A guided tour, Graduate Texts in Mathematical Physics, II. International Press, Cambridge, MA, 1993.

[49] V. A. Smirnov, Renormalization and Asymptotic Expansions, Birkhäuser Verlag, Basel, 1991.

[50] L. Solomon, A Mackey formula in the group ring of a Coxeter group, J. Algebra 41 (1976) 255-268.

[51] G. 't Hooft, Dimensional regularization and the renormalization group, Nucl. Phys. B 41 (1973) $455-468$.

[52] A. N. Vasilev, The field theoretic renormalization group in critical behavior theory and stochastic dynamics, Chapman \& Hall/CRC, Boca Raton, FL, 2004.

[53] W. Zimmermann, Convergence of Bogoliubov's method of renormalization in momentum space, Commun. Math. Phys. 15 (1969) 208-234.

I.H.É.S., Le Bois-Marie, 35, Route de Chartres, F-91440 Bures-Sur-Yvette, France

E-mail address: kurusch@ihes.fr

$U R L:$ http://www.th.physik.uni-bonn.de/th/People/fard/

Departamento de Física Teórica I, Universidad Complutense, Madrid 28040, Spain and Departamento de Física, Universidad de Costa Rica, San Pedro 2060, Costa Rica

Laboratoire J.-A. Dieudonné UMR 6621, CNRS, Parc Valrose, 06108 Nice Cedex 02, France

E-mail address: patras@math.unice.fr

$U R L$ : www-math.unice.fr/ patras 